\title{
A Mixed, Unified Forward/Inverse Framework for Earthquake Problems: Fault Implementation and Coseismic Slip Estimate
}

\author{
Simone Puel ${ }^{1,2}$, Eldar Khattatov ${ }^{3}$, Umberto Villa ${ }^{4}$, Dunyu Liu ${ }^{2}$, \\ Omar Ghattas ${ }^{1,3,5}$, and Thorsten W. Becker ${ }^{1,2,3}$ \\ ${ }^{1}$ Department of Geological Sciences, Jackson School of Geosciences, The University of Texas at Austin \\ ${ }^{2}$ Institute for Geophysics, Jackson School of Geosciences, The University of Texas at Austin \\ ${ }^{3}$ Oden Institute for Computational Engineering and Sciences, The University of Texas at Austin \\ ${ }^{4}$ Electrical and Systems Engineering, Washington University in St. Louis \\ ${ }^{5}$ Walker Department of Mechanical Engineering, The University of Texas at Austin
}

This manuscript is a preprint uploaded to EarthArXiv. This preprint has been
submitted for publication in Geophysical Journal International and has not yet
been peer-reviewed. We welcome feedback, discussion and comments at any
time. Feel free to get in touch with one of the authors.

Corresponding author: Simone Puel

Email:spuel@utexas.edu 


\title{
A Mixed, Unified Forward/Inverse Framework for Earthquake Problems: Fault Implementation and Coseismic Slip Estimate
}

\author{
S. Puel ${ }^{1,2 \star}$, E. Khattatov ${ }^{3}$, U. Villa ${ }^{4}$, D. Liu ${ }^{2}$, O. Ghattas ${ }^{1,3,5}$ and T. W. Becker ${ }^{1,2,3}$ \\ ${ }^{1}$ Department of Geological Sciences, Jackson School of Geosciences, The University of Texas at Austin, Austin, TX 78712, USA \\ ${ }^{2}$ Institute for Geophysics, Jackson School of Geosciences, The University of Texas at Austin, Austin, TX 78712, USA \\ ${ }^{3}$ Oden Institute for Computational Engineering and Sciences, The University of Texas at Austin, Austin, TX 78712, USA \\ ${ }^{4}$ Electrical and Systems Engineering, Washington University in St. Louis, St. Louis, MO 63112, USA \\ ${ }^{5}$ Walker Department of Mechanical Engineering, The University of Texas at Austin, Austin, TX 78712, USA
}

\section{SUMMARY}

We introduce a new finite-element (FE) based computational framework to solve forward and inverse elastic deformation problems for earthquake faulting via the adjoint method. Based on two advanced computational libraries, FENICS and HIPPYLIB for the forward and inverse problems, respectively, this framework is flexible, transparent, and easily extensible. We represent a fault discontinuity through a mixed FE elasticity formulation, which approximates the stress with higher order accuracy and exposes the prescribed slip explicitly in the variational form without using conventional split node and decomposition discrete approaches. This also allows the first order optimality condition, i.e., the vanishing of the gradient, to be expressed in continuous form, which leads to consistent discretizations of all field variables, including the slip. We show comparisons with the standard, pure displacement formulation and a model containing an in-plane mode II crack, whose slip is prescribed via the split node technique. We demonstrate the poten- 
tial of this new computational framework by performing a linear coseismic slip inversion through adjoint-based optimization methods, without requiring computation of elastic Green's functions. Specifically, we consider a penalized least squares formulation, which in a Bayesian setting - under the assumption of Gaussian noise and prior-reflects the negative $\log$ of the posterior distribution. The comparison of the inversion results with a standard, linear inverse theory approach based on Okada's solutions shows analogous results. Preliminary uncertainties are estimated via eigenvalue analysis of the Hessian of the penalized least squares objective function. Our implementation is fully open-source and Jupyter notebooks to reproduce our results are provided. The extension to a fully Bayesian framework for detailed uncertainty quantification and non-linear inversions, including for heterogeneous media earthquake problems, will be analyzed in a forthcoming paper.

Key words: Seismic cycle; Inverse theory; Numerical approximations and analysis; Earthquake source observations; Kinematics of crustal and mantle deformation.

\section{INTRODUCTION}

Over the past few decades, revolutionary advances in space geodesy and new seismic networks have allowed scientists to quantitatively measure deformation at depth and surface displacements before, during, and after an earthquake. This technological progress has led to new observations regarding deviations from the simple concept of stationary interseismic loading, followed by instantaneous coseismic and short-term postseismic periods. For instance, the occurrence of slow-slip events (e.g. Ito et al. 2013; Uchida \& Matsuzawa 2013) and long-transient surface deformation (Ozawa et al. 2012; Mavrommatis et al. 2014; Yokota \& Koketsu 2015), crustal stress changes (e.g. Yoshida et al. 2012; Becker et al. 2018) and decrease in seismic $b$-value (Nanjo et al. 2012) were observed around the rupture area before the 2011 M9 Tohoku-oki earthquake in Japan (Uchida \& Bürgmann 2021), as well as a response of seismic velocities at crustal depths right after it (Wang et al. 2019, 2021). The mechanisms responsible for these phenomena, visible also in other subduction zones (e.g., Dragert et al. 2001; Ruiz et al. 2014; Wallace et al. 2016; Wallace 2020), and whether the preseismic observations may be considered precursors of the megathrust event are still under debate (Pritchard et al. 2020).

\footnotetext{
^ Email: spuel@utexas.edu
} 
Computational modeling can help to integrate these observational data into numerical models to better investigate the physics of these earthquake processes (e.g., Bartlow et al. 2014; Williams \& Wallace 2015; Erickson et al. 2020; Im et al. 2020; Sun et al. 2020). For example, Im et al. (2020) used rate-and-state friction simulations and experimental data to analyze the nature of slow slip events as a rate-dependent transition from rate-weakening frictional sliding at low slip rates to more ratestrengthening behavior at higher slip rates. Sun et al. (2020), instead, used field observations and the finite-element (FE) code SULEC (Ellis et al. 2015) to study the effect of mechanical and hydrological processes in the forearc deformation in response to seamount subduction.

Many of the models in use are by necessity designed to focus on one or a few processes of the earthquake system. The development of more community-driven efforts, such as PYLITH (Aagaard et al. 2013) and RELAX (Barbot 2014), have led to more versatile codes that can capture a wider range of the earthquake cycle, from spontaneous rupture propagation to interseismic and postseismic deformation. Some of the advantages of these publicly available software are the presence of well-documented user manuals, tutorials and developers-users seminars, code sharing, and benchmark comparisons. However, although increasingly well benchmarked and flexible, these codes can, in practice, suffer from a lack of transparency and flexibility in terms of implementing different physics. The governing equations and solver strategies may be hard-coded into the software, requiring moderate to advanced knowledge of computer science to implement multi-physics coupled problems. Commercial software, such as ABAQUS (Smith 2009) and COMSOL MULTIPHYSICS (Multiphysics 1998), can be deficient in transparency but allow the user to choose among a wide range of material rheologies and solver options. Both open source and commercial approaches have limitations when combining forward and inverse approaches is the objective, as is the case when the goal is to infer fault and host rock rheology in a heterogeneous medium, a key problem given uncertainties about effective mechanical properties and material parameters, for example in the megathrust context.

Recent open-source advanced libraries have overcome the issues of transparency and flexibility by providing access to most of the low-level numerical functionality. For instance, the scientific computing libraries PETSC (Balay et al. 1997, 2020) and TRILINOS (Heroux et al. 2005) allow the user to easily test different algorithms and solvers options. Similarly, numerical frameworks like DEAL.II (Bangerth et al. 2007), FENICS (Logg \& Wells 2010; Logg et al. 2012), and FIREDRAKE (Rathgeber et al. 2016) provide tools for the FE discretization in which new equations can be input via variational forms. In particular, FENICS and FIREDRAKE provide an expressive declaration of any PDE via Python using the Unified Form Language (Alnæs et al. 2014). This allows description of weak forms of equations in a simple and efficient way, and distribution of solver approaches as JUPYTER notebooks (Project Jupyter 2021) for research and teaching objectives. 
Early efforts to build on these advanced libraries have demonstrated high degree of flexibility in the problem description (Simpson et al. 2010; Wilson et al. 2014, 2017; Spiegelman et al. 2016; McCormack \& Hesse 2018). For example, TERRAFERMA (Wilson et al. 2014; Spiegelman et al. 2016; Wilson et al. 2017) integrates the functionality of the FENICS, PETSC, and SPUD (Ham et al. 2009) libraries into an interface where the user can choose among different computational and solver options, while having the ability to write its own governing equations. This makes the extension to new non-linear multi-physics coupled problems relatively easy to implement. In addition, sharing the same framework, each model is self-documenting, making the code more reusable. However, the additional SPUD layer also removes some of the transparency of accessing FENICS more directly, and TERRAFERMA is designed to only solve the system of PDEs in a forward sense.

Finally, a desired feature of many such frameworks is the capability to extract information from observations via an inverse problem and quantify the associated model uncertainties. Although there are many available inversion packages for the solution of the inverse problem (e.g., Adams et al. 2009; Farrell et al. 2013; Parno et al. 2014; Ruthotto et al. 2017; McDougall et al. 2017; Tong 2017), none of them provide the flexibility and discretization, optimization, and statistical tools to efficiently solve scalable PDE-based deterministic and Bayesian inverse problems such as those encountered for the earthquake problem (Villa et al. 2021).

Here, we therefore exploit an open-source framework based on two advanced libraries, FENICS and HIPPYLIB (Villa et al. 2016, 2018, 2021), to incorporate both forward and inverse capabilities within the same computational architecture. The underlying algorithms have been successfully applied to solve complex PDE-based inverse problems such as in seismic wave propagation (Bui-Thanh et al. 2012, 2013), ice sheet flow (Petra et al. 2014; Isaac et al. 2015; Babaniyi et al. 2021), poroelasticity (Hesse \& Stadler 2014; Alghamdi et al. 2020), mantle convection (Worthen et al. 2014), and turbulent flow (Chen et al. 2019), but rarely to solve earthquake problems (McCormack et al. 2018). For instance, McCormack et al. (2018) used GPS surface deformation measurements to infer the coseismic slip distribution of the 2012 M7.6 Costa Rica earthquake. However, they only considered the overriding plate by inverting for the slip boundary conditions, without considering the entire fault system. A crucial component to model, for example, the seismic cycle and slip behavior (e.g., slow slip, slab-overriding plate interactions, fluid flow, etc.) is the inclusion of the fault discontinuity.

We propose a new implementation of the fault within a mixed FE elastic formulation, which exposes the prescribed slip explicitly in the variational form, without using split node (Melosh \& Raefsky 1981) or domain discrete decomposition (e.g. Aagaard et al. 2013) approaches. We use the FENICSHIPPYLIB framework to show how this discretization of the governing equations can be easily implemented and used to solve the elastic forward problem. Moreover, we compute a coseismic slip 
inversion using adjoint-based optimization methods without a priori fault discretization and Green's function computations. We compare the results to the standard linear method based on Okada's analytic fault segment solutions (Okada 1985, 1992), and estimate preliminary model uncertainties from spectrum decomposition. In a subsequent paper, we will extend this linear inversion to a fully Bayesian framework for detailed uncertainty quantification and provide non-linear inversions, e.g., for heterogeneous material parameters, for earthquake type problems.

This manuscript is structured as follows. We first present a brief description of the forward-inverse framework in Section 2, and then compare the new fault implementation within the mixed FE elastic formulation to the standard displacement method and to the split node technique in Section 3. Next, we address the common coseismic slip problem as an example application and compare our inversion results to the classic linear approach using Green's functions (Section 4). We conclude in Section 5 by discussing capabilities and limitations of our approach.

\section{FENICS-HIPPYLIB FRAMEWORK}

Our open-source forward-inverse modeling framework is based on two advanced numerical libraries, FENICS and HIPPYLIB. FENICS (Logg \& Wells 2010; Logg et al. 2012) is a high-level parallel FE collection of software components for automated and efficient solution of PDEs. It includes several libraries for the FE discretization, assembly and solution of linear and non-linear systems of equations. In FENICS, any PDE can be explicitly and easily expressed in variational form using the Unified Form Language (Alnæs et al. 2014) Python library. This makes a problem coded in this framework transparent, reproducible, flexible for multi-physics formulations, and easy to implement. The variational forms of these equations can then be automatically discretized, converted, and assembled into lowlevel C++ codes using the FENICS form compiler (Kirby \& Logg 2006) and the high performance library DOLFIN (Logg \& Wells 2010). The latter provides the user interface and integrates all other computational components, communicating with external libraries such as PETSC (Balay et al. 1997, 2020) and TRILINOS (Heroux et al. 2005) for the numerical linear algebra, SCOTCH (Pellegrini 2008) for the mesh partitioning, and MPI (Gropp et al. 1999) and OpenMP (Dagum \& Menon 1998) for parallel computing. FENICS is well tested and benchmarked through several available demos and applications in the Earth sciences (e.g., Vynnytska et al. 2013; Tosi et al. 2015; Rhebergen et al. 2015; Damiani et al. 2020; Haagenson et al. 2020). For instance, Vynnytska et al. (2013) provided 2D/3D benchmarks for mantle convection problems, and Wilson \& Spiegelman (2016) conducted a wide range of geodynamic benchmarks using TERRAFERMA, including for subduction zone thermal structure (van Keken et al. 2008).

This advanced framework can inter-operate with the HIPPYLIB package (Villa et al. 2016, 2018, 
2021). Built on FENICS and PETSC for the discretization of the PDEs and scalable linear algebra operations and solvers, respectively, this library implements state-of-the-art scalable adjoint-based algorithms for PDE-based deterministic and Bayesian inverse problems. In HIPPYLIB, derivative information-i.e. gradients and actions of the second derivative of objective functions (Hessian)—are efficiently computed using the adjoint method while leveraging the automated symbolic differentiation and assembly of variational forms in FENICS. These are essential ingredients not only for the solution of the deterministic inverse problem but also for uncertainty quantification.

HIPPYLIB preserves all of the flexibility of the underlying libraries, allowing solution of linear and non-linear and stationary and time-dependent PDE-based systems of equations. It provides a collection of functions for deterministic and Bayesian solution of inverse problems, accelerated by adjoint-based gradient and Hessian operations. While in a deterministic inversion the result is found by a least squares minimization for the "best" model parameters, a Bayesian framework provides a posterior distribution of likely values within a range, thereby quantifying uncertainties and trade-offs in information. Algorithms to solve linear and non-linear deterministic inverse problems in HIPPYLIB make use of common kernels, such as randomized singular value decomposition (SVD) methods (Villa et al. 2021). In the linear case, for example, the solution of the deterministic inverse problem is found using conjugate gradients (CG), while for the Bayesian inverse problem, the posterior is Gaussian with mean given by the solution of the deterministic problem and covariance by the inverse of the Hessian. In the case of non-linear inverse problems, the deterministic inversion is solved by use of inexact Newton-CG, while Bayesian solution is computed using geometric Markov chain Monte Carlo (MCMC) methods (Beskos et al. 2017), which employ Hessian information to accelerate sampling.

Issues such as high-dimensionality $\left(\mathcal{O}\left(10^{6}\right)\right.$ parameters) of large non-linear geophysical problems, highly concentrated posterior distributions (Baumann et al. 2014; Baumann \& Kaus 2015; Gallovič et al. 2019), and the slow convergence of Monte Carlo methods have made Bayesian inversion for complex problems intractable using methods such as black-box MCMC. HIPPYLIB efficiently overcomes these challenges by exploiting the intrinsic low dimensionality of the parameter-to-observable map of the problem (Flath et al. 2011; Bui-Thanh et al. 2012; Isaac et al. 2015; Wang et al. 2018; Chen et al. 2019), and by exploiting posterior geometry via adjoint-based gradient and low-rank Hessian information (e.g., Bashir et al. 2008; Beskos et al. 2017; Martin et al. 2012; Petra et al. 2014; Bui-Thanh \& Ghattas 2015; Alexanderian et al. 2016). These techniques require a number of forward model solves that is independent of the parameter or data dimension (as opposed to, for example, gradient-only or derivative-free methods), and depend only on the intrinsic information contained in the data about the model (e.g. Bui-Thanh et al. 2012, 2013; Isaac et al. 2015). 


\section{THE FORWARD ELASTIC PROBLEM}

Let us consider a connected and bounded domain $\Omega \subset \mathbb{R}^{d}, d=2,3$ with boundaries $\partial \Omega=\Gamma_{D} \cup \Gamma_{N}$, where $\Gamma_{D}$ and $\Gamma_{N}$ represent Dirichlet and Neumann boundaries, respectively. The linear spaces of vectors, matrices, symmetric matrices and skew-symmetric matrices are denoted by $\mathbb{V}, \mathbb{M}, \mathbb{S}$ and $\mathbb{K}$, respectively.

Given a vector field $\boldsymbol{f}(\boldsymbol{x})$ for each point $\boldsymbol{x} \in \Omega$ indicating body forces, the equation of linear elasticity in the Hellinger-Reissner form seeks to find the stress $\boldsymbol{\sigma}$ and displacement $\boldsymbol{u}$ which satisfy the constitutive and the linear momentum equations, including the boundary conditions (e.g., Arnold 1990; Arnold et al. 2007):

$$
\begin{cases}\boldsymbol{A} \boldsymbol{\sigma}=\boldsymbol{\varepsilon}(\boldsymbol{u}) & \text { in } \Omega, \\ \nabla \cdot \boldsymbol{\sigma}=-\boldsymbol{f} & \text { in } \Omega \\ \boldsymbol{u}=\boldsymbol{u}_{0} & \text { on } \Gamma_{D} \\ \boldsymbol{\sigma} \cdot \mathbf{n}=\boldsymbol{t} & \text { on } \Gamma_{N}\end{cases}
$$

where $\boldsymbol{A}=\boldsymbol{A}(\boldsymbol{x})$ is the fourth-order elastic compliance tensor, which is a symmetric and positive definite linear operator $\mathbb{M} \rightarrow \mathbb{M}$ describing the material properties of the medium. $\varepsilon(\boldsymbol{u})=\operatorname{sym}(\nabla \boldsymbol{u})=$

$\frac{1}{2}\left(\nabla \boldsymbol{u}+(\nabla \boldsymbol{u})^{T}\right)$ is the strain tensor, which is the symmetric part of the gradient deformation tensor, $\boldsymbol{u}_{0}$ is the imposed displacement values at the boundaries, $\mathbf{n}$ denotes the outward unit normal of $\partial \Omega$, and $\boldsymbol{t}$ is the traction. In the case of an homogeneous and isotropic elastic material, the compliance $\boldsymbol{A}(\boldsymbol{x})$ depends only on the two Lamé coefficients, the shear modulus $\mu$ and $\lambda$ :

$\boldsymbol{A} \boldsymbol{\sigma}=\frac{1}{2 \mu}\left(\boldsymbol{\sigma}-\frac{\lambda}{2 \mu+d \lambda} \operatorname{tr}(\boldsymbol{\sigma}) \boldsymbol{I}\right)$,

where $\boldsymbol{I}$ is the $d \times d$ identity matrix, and $\operatorname{tr}(\boldsymbol{\sigma})$ is the trace of the stress tensor. The formula above relies on the symmetry of the stress tensor and the invertibility of the stress-strain relation (e.g. Rognes \& Winther 2010).

In this work, we are interested in simulating the cosesmic slip along a fault plane $\Gamma_{F}$ in $\Omega$, as shown in Fig. 1. For a fault discontinuity $\Gamma_{F}$, let $\mathbf{n}^{+}$and $\mathbf{n}^{-}$be the two unit normal vector fields on $\Gamma_{F}$ with opposite directions $\left(\mathbf{n}^{+}=-\mathbf{n}^{-}=\mathbf{n}_{\Gamma_{F}}\right)$. For example, $\mathbf{n}^{+}$represents the outward unit normal vector from the " + " side pointing towards the negative side of the fault. Then using the above notation, we write the interface conditions at the fault interface as

$$
\begin{cases}\llbracket T\left(\mathbf{n}_{\Gamma_{F}}\right) \boldsymbol{u} \rrbracket=s & \text { on } \Gamma_{F}, \\ \llbracket \boldsymbol{u} \cdot \mathbf{n}_{\Gamma_{F}} \rrbracket=0 & \text { on } \Gamma_{F}, \\ \llbracket \boldsymbol{\sigma} \cdot \mathbf{n}_{\Gamma_{F}} \rrbracket=0 & \text { on } \Gamma_{F},\end{cases}
$$

where $\llbracket \phi \rrbracket=\phi^{+}-\phi^{-}$is the jump operator, and $T\left(\mathbf{n}_{\Gamma_{F}}\right)$ is a tangent operator which allows to take 


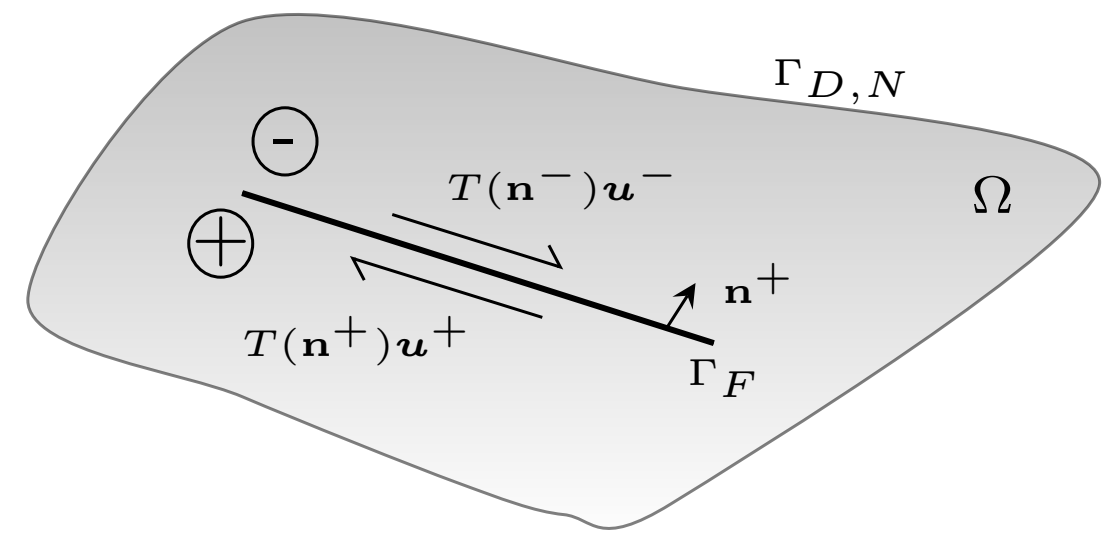

Figure 1. Schematic diagram of the fault $\Gamma_{F}$ within the domain $\Omega$. "+" and "-_" signs indicate the positive and negative sides of the discontinuity, respectively, and $\mathbf{n}$ is the outward unit normal vector field on $\Gamma_{F}$. The fault slip is described as a jump in the displacement field $\boldsymbol{u}$ across the fault.

the component of the displacement $\boldsymbol{u}$ parallel to the fault plane $\Gamma_{F}$. We define the vector $T(\mathbf{n})=$ $\left(n_{2},-n_{1}\right)$ in $2 \mathrm{D}$ and in $3 \mathrm{D}$ the $2 \times 3$ matrix as

$T(\mathbf{n})=\left(\begin{array}{ccc}0 & -n_{3} & n_{2} \\ n_{2}^{2}+n_{3}^{2} & -n_{1} n_{2} & -n_{1} n_{3}\end{array}\right)$,

where these two expressions can be found by decomposing the unit normal over the basis. In $2 \mathrm{D}$ we look for a vector orthogonal to a given normal, and in 3D we first get a plane and then find an orthogonal pair.

To derive a variational formulation of the strong form in eq. (1) with the fault interface condition stated in eq. (3), we first introduce the Lagrange multiplier $\boldsymbol{r}=\operatorname{skew}(\nabla \boldsymbol{u})=\frac{1}{2}\left(\nabla \boldsymbol{u}-(\nabla \boldsymbol{u})^{T}\right)$ from the space of skew-symmetric matrices $\mathbb{K}$, which has a physical meaning of rotation (Fraeijs de Veubeke 1975), and substitute $\varepsilon(\boldsymbol{u})=\nabla \boldsymbol{u}-\boldsymbol{r}$. Then, we construct the variational form by taking the dot product of eq. (1) with weighting functions $\tau \in \Sigma, \omega \in W$, and $\xi \in \Xi$, and setting the integral over $\Omega$ equal to zero:

$$
\begin{aligned}
\int_{\Omega} \boldsymbol{A} \boldsymbol{\sigma}: \tau d \boldsymbol{x}-\int_{\Omega} \nabla \boldsymbol{u} \cdot \tau d \boldsymbol{x}+\int_{\Omega} \operatorname{as}(\tau) \cdot \boldsymbol{r} d \boldsymbol{x}=0 & \forall \tau \in \Sigma \\
\int_{\Omega}(\nabla \cdot \boldsymbol{\sigma}) \cdot \omega d \boldsymbol{x}+\int_{\Omega} \boldsymbol{f} \cdot \omega d \boldsymbol{x}=0 & \forall \omega \in W, \\
\int_{\Omega} \operatorname{as}(\boldsymbol{\sigma}) \cdot \xi d \boldsymbol{x}=0 & \forall \xi \in \Xi
\end{aligned}
$$

where $\tau, \omega, \xi$ are the test functions for stress, displacement and rotation, respectively, and the spaces are defined as $\Sigma=\left\{\tau \in H(\nabla \cdot, \Omega \mathbb{M}): \tau \mathbf{n}=0\right.$ on $\Gamma_{N}, \llbracket \tau \mathbf{n} \rrbracket=0$ on $\left.\Gamma_{F}\right\}, W=L^{2}\left(\Omega, \mathbb{R}^{d}\right)$, $\Xi=L^{2}(\Omega, \mathbb{K})$, which represent the space of square-integrable matrices fields with square-integrable 
divergence satisfying the traction boundary conditions, and the spaces of all square integrable vector fields (Arnold 1990). The restriction on the stress space along $\Gamma_{F}$ is effectively satisfied when the fault is resolved by the computational grid. The last equation of eq. (5) is necessary in order to enforce the symmetry of the stress tensor weakly (Arnold et al. 1984a; Stenberg 1988; Farhloul \& Fortin 1997; Arnold et al. 2007; Boffi et al. 2009; Cockburn et al. 2010). We define the asymmetry operator $\operatorname{as}(\tau)=\left(\tau_{12}-\tau_{21}\right)$ in $2 \mathrm{D}$ and $\operatorname{as}(\tau)=\left(\tau_{32}-\tau_{23}, \tau_{31}-\tau_{13}, \tau_{21}-\tau_{12}\right)^{T}$ in 3D. Note that the Lagrange multiplier $\boldsymbol{r}$ is a scalar and vector field in 2D and 3D, respectively.

The integration by parts of the non-conforming term in the first equation gives

$\int_{\Omega} \nabla \boldsymbol{u} \cdot \tau d \boldsymbol{x}=-\int_{\Omega} \boldsymbol{u} \cdot(\nabla \cdot \tau) d \boldsymbol{x}+\int_{\partial \Omega} \boldsymbol{u}_{0} \cdot(\tau \mathbf{n}) d s+\int_{\Gamma_{F}} \llbracket \boldsymbol{u} \cdot(\tau \mathbf{n}) \rrbracket d S$,

where $d s$ and $d S$ represent the integration of the integrand over the external and internal boundaries, respectively. We recognize that the traction boundary condition $(\sigma \cdot \mathbf{n}=\boldsymbol{t})$ becomes essential, hence it has to be imposed a priori onto the function space where the stress tensor is sought, while the displacement boundary condition $\left(\boldsymbol{u}=\boldsymbol{u}_{0}\right)$ arises naturally from the weak form derivation. Decomposing the last term in eq. (6) into its normal and tangential (relative to the fault $\Gamma_{F}$ ) components results in

$$
\begin{aligned}
\int_{\Gamma_{F}} \llbracket \boldsymbol{u} \cdot(\tau \mathbf{n}) \rrbracket d S & =\int_{\Gamma_{F}} \llbracket T(\mathbf{n}) \boldsymbol{u} \cdot T(\mathbf{n})(\tau \mathbf{n}) \rrbracket d S+\int_{\Gamma_{F}} \boldsymbol{u} \cdot \mathbf{n} \llbracket(\tau \mathbf{n}) \cdot \mathbf{n} \rrbracket d S \\
& =\int_{\Gamma_{F}} \boldsymbol{s} \cdot T(\mathbf{n})(\tau \mathbf{n}) d S,
\end{aligned}
$$

where we have used the slip definition (eq. 3) and the fact that $\tau \in \Sigma$ to obtain the last equality. Hence, the variational formulation of the elasticity equation in this mixed form reads: seek $(\boldsymbol{\sigma}, \boldsymbol{u}, \boldsymbol{r}) \in$ $\Sigma \times W \times \Xi$ such that

$$
\begin{aligned}
& \int_{\Omega} \boldsymbol{A} \boldsymbol{\sigma}: \tau d \boldsymbol{x}+\int_{\Omega} \boldsymbol{u} \cdot(\nabla \cdot \tau) d \boldsymbol{x}+\int_{\Omega} \operatorname{as}(\tau) \cdot \boldsymbol{r} d \boldsymbol{x}=\int_{\Gamma_{F}} \boldsymbol{s} \cdot\left[T\left(\mathbf{n}^{+}\right)\left(\tau^{+} \mathbf{n}^{+}\right)\right] d S \quad \forall \tau \in \Sigma, \\
& \int_{\Omega}(\nabla \cdot \boldsymbol{\sigma}) \cdot \omega d \boldsymbol{x}=-\int_{\Omega} \boldsymbol{f} \cdot \omega d \boldsymbol{x} \quad \forall \omega \in W, \\
& \int_{\Omega} \operatorname{as}(\boldsymbol{\sigma}) \cdot \xi d \boldsymbol{x}=0 \quad \forall \xi \in \Xi
\end{aligned}
$$

where we use the superscript "+" to indicate the side of the fault where the slip is prescribed (Fig. 1). In this scenario, the slip $s$ will be negative for a thrust fault and positive for a normal movement. Vice-versa, we could also write a negative superscript for the unit normal, but in this case we would need to take a positive sign of the slip to indicate a reverse movement.

We will see in the next section how the fault problem formulation in eq. (8) can be implemented by using a stable pair of function spaces to discretize the displacement field with discontinuous piecewise polynomial elements, thus allowing relative motions between two adjacent cells. This approach, which naturally arises from the integration by parts in the derivation of the variational formulation, is 
contrasted with the split node (Melosh \& Raefsky 1981) and the decomposition (e.g. Aagaard et al. 2013) techniques. While in the first method the slip is explicitly prescribed on both sides of the fault through a modification of the local force vector, in the decomposition approach the slip is imposed as double couple point sources via a Lagrange multiplier. Our approach differs from the methods above in the sense that the slip is prescribed, at the continuum level, directly as a constraint in the displacement field, without applying a proper traction or requiring local modifications of the force vector. In addition, our approach does not introduce additional unknown variables at the fault nodes as for example PyLith (Aagaard et al. 2017) does, which generally requires particular techniques for the matrix solution (Aagaard et al. 2013). Another advantage of our approach is that the slip vector appears explicitly in the weak form, which allows derivation of the gradient for the inverse problem at the continuum level, leading to consistent discretizations of all field variables, and inversion for the slip distribution without having to compute elastic Green's functions (Section 4).

\subsection{Mixed Finite Element Method}

Next, we present the mixed FE approximation of eq. (8). There are several studies that analyze the choice of stable finite-element spaces $\Sigma_{h}, W_{h}$, and $\Xi_{h}$ to discretize stress, displacement and rotation, respectively (e.g., Arnold et al. 2007; Falk 2008; Cockburn et al. 2010; Rognes \& Winther 2010; Ambartsumyan et al. 2020). The most common choices are those introduced by Arnold et al. (2007), where the lowest order elements are the union of linear vector polynomials with continuity of normal components over the element facets for the stress (Brezzi et al. 1985; Nédélec 1986), and piecewise discontinuous constants for the displacement and the rotation (Fig. 2, top panel).

For an arbitrary polynomial degree $k$ and considering a 2D/3D finite-element triangulation $\mathcal{T}_{h}$ of the domain $\Omega$, the $k$ 'th order elements for the elasticity equation are

$\Sigma_{h}=B D M_{k}\left(\mathcal{T}_{h}, \mathbb{V}\right) ; \quad W_{h}=D G_{k-1}\left(\mathcal{T}_{h}, \mathbb{V}\right) ; \quad \Xi_{h}=D G_{k-1}\left(\mathcal{T}_{h}, \mathbb{K}\right)$,

where $B D M_{k}$ represent the $k$ 'th order of Brezzi-Douglas-Marini elements (Brezzi et al. 1985) which are vector polynomials with continuity of normal components over inter-element facets (Fig. 2). The choice to use piecewise discontinuous Galerkin elements $D G_{k-1}$ to discretize the displacement vector field allows to prescribe relative motion between two adjacent cells on the fault plane.

Therefore, the mixed FE approximation of eq. (8) can be written as: find $\left(\boldsymbol{\sigma}_{h}, \boldsymbol{u}_{h}, \boldsymbol{r}_{h}\right) \in \Sigma_{h} \times$ 

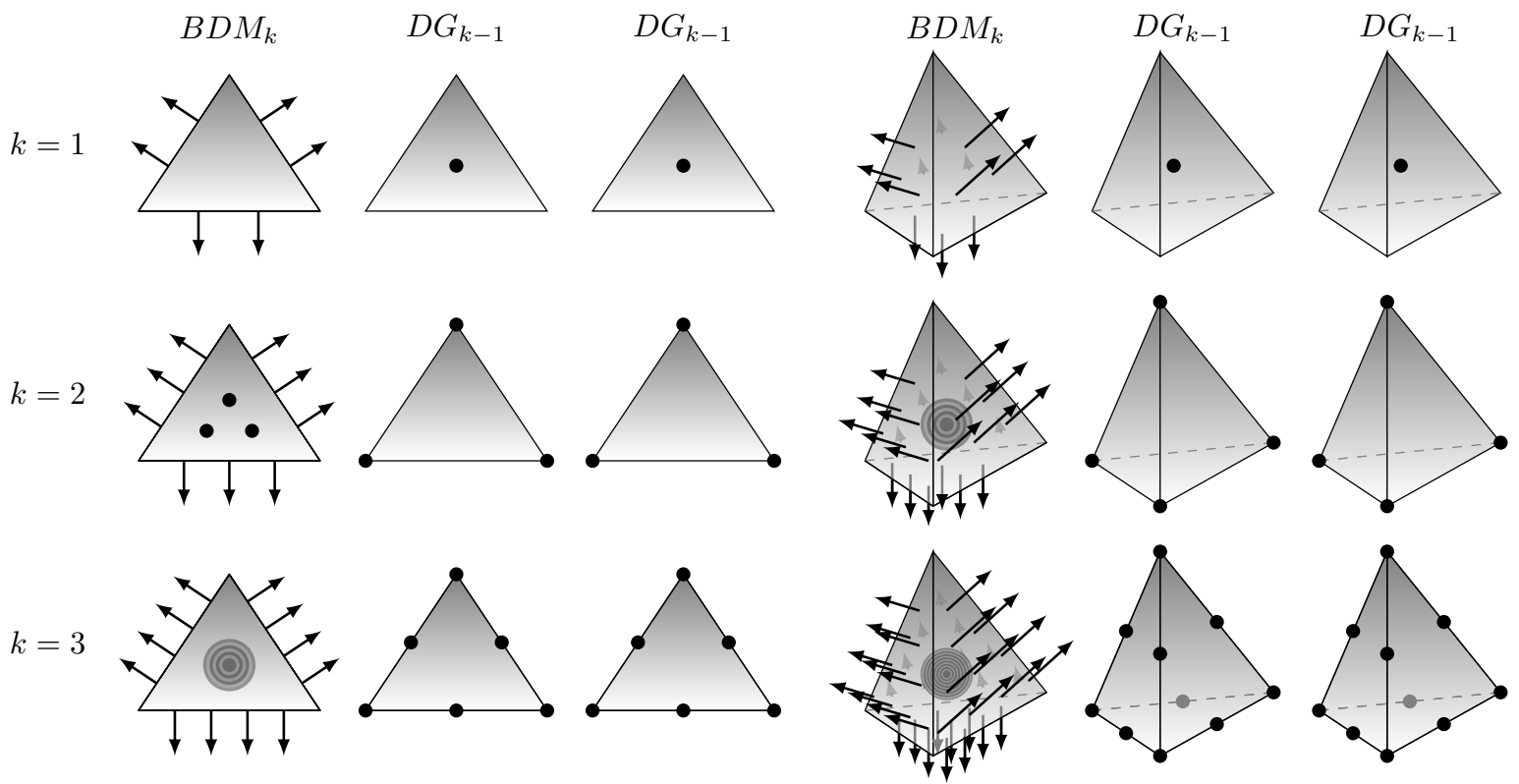

Figure 2. First, second and third degree $k^{t h}$ of $B D M_{k} \times D G_{k-1} \times D G_{k-1}$ elements for the discretization of stress, displacement and rotation, respectively. Left: Brezzi-Douglas-Marini $\left(B D M_{k}\right)$ and Discontinuous Lagrange ( $\left.D G_{k-1}\right)$ elements for triangles (2D). Right: same as above, but for tetrahedra (3D). Black dots indicate the degrees-of-freedom (DOFs) within each element. Arrows normal to an edge or a face denote DOFs associated to normal components of vector fields along that edge or face. Gray circles and dots of $B D M$ elements indicate bubble functions, that is functions with vanishing normal trace on the boundary of the element. For the discontinuous Lagrange elements, all the DOFs are internal to the elements.

$W_{h} \times \Xi_{h}$ such that

$$
\begin{aligned}
\int_{\Omega_{h}} \boldsymbol{A} \boldsymbol{\sigma}_{h}: \tau d \boldsymbol{x}+\int_{\Omega_{h}} \boldsymbol{u}_{h} \cdot(\nabla \cdot \tau) d \boldsymbol{x}+\int_{\Omega_{h}} \operatorname{as}(\tau) \cdot \boldsymbol{r}_{h} d \boldsymbol{x} & =\int_{\Gamma_{h, F}} \boldsymbol{s} \cdot\left[T\left(\mathbf{n}^{+}\right)\left(\tau^{+} \mathbf{n}^{+}\right)\right] d S \quad \forall \tau \in \Sigma_{h}, \\
\int_{\Omega_{h}}\left(\nabla \cdot \boldsymbol{\sigma}_{h}\right) \cdot \omega d \boldsymbol{x} & =-\int_{\Omega_{h}} \boldsymbol{f} \cdot \omega d \boldsymbol{x} \quad \forall \omega \in W_{h}, \\
\int_{\Omega_{h}} \operatorname{as}\left(\boldsymbol{\sigma}_{h}\right) \cdot \xi d \boldsymbol{x} & =0 \quad \forall \xi \in \Xi_{h},
\end{aligned}
$$

where $\Omega_{h}$ is the discretized domain. The mesh conforms to the fault geometry, that is $\Gamma_{h, F}$ is the union of facets which align with the fault geometry in the triangulation of $\Omega_{h}$. This system of equations has a unique solution both on continuous and discrete levels. The choice of the spaces $\Sigma_{h}, W_{h}$, and $\Xi_{h}$ in eq. (10) provide stable finite-element approximations (e.g., Arnold et al. 2007), which satisfy the stability conditions in the Brezzi's theory of mixed methods (Brezzi 1974). Lastly, the system possesses the same order accuracy for all variables in their corresponding norms (e.g., Arnold et al. 2007; 


\section{S. Puel et al.}

Cockburn et al. 2010; Ambartsumyan et al. 2020), and advantage over the displacement formulation, which yields one order lower stresses.

\subsubsection{Solution of Saddle-Point Type Systems}

In matrix-vector form, the system $\mathbf{A x}=\mathbf{b}$ of eq. (8) can be written as

$$
\left(\begin{array}{ccc}
A_{\sigma \sigma} & A_{\sigma u}^{T} & A_{\sigma r}^{T} \\
A_{\sigma u} & 0 & 0 \\
A_{\sigma r} & 0 & 0
\end{array}\right)\left(\begin{array}{l}
\boldsymbol{\sigma} \\
\boldsymbol{u} \\
\boldsymbol{r}
\end{array}\right)=\left(\begin{array}{c}
M_{F} \boldsymbol{s} \\
-M_{\Omega} \boldsymbol{f} \\
0
\end{array}\right),
$$

where: $\left(A_{\sigma \sigma}\right)_{i j}=\int_{\Omega} \boldsymbol{A} \tau_{j}: \tau_{i} d \boldsymbol{x},\left(A_{\sigma u}\right)_{i j}=\int_{\Omega}\left(\nabla \cdot \tau_{j}\right) \cdot \omega_{i} d \boldsymbol{x}$, and $\left(A_{\sigma r}\right)_{i j}=\int_{\Omega} \tau_{j} \cdot \xi_{i} d \boldsymbol{x}$, where $\left\{\tau_{i}\right\}_{i},\left\{\omega_{i}\right\}_{i},\left\{\xi_{i}\right\}_{i}$ represent the FE basis of the spaces $\Sigma_{h}, W_{h}$, and $Q_{h}$. The mass matrices $\left(M_{F}\right)_{i j}=\int_{\Gamma_{F}} \psi_{j}\left[T\left(\mathbf{n}^{+}\right)\left(\tau_{i}^{+} \mathbf{n}^{+}\right)\right] d S$ and $\left(M_{\Omega}\right)_{i j}=\int_{\Omega} \omega_{i} \cdot \omega_{j} d \boldsymbol{x}$ represent the FE basis of the spaces to discretize the slip $\left(\psi_{j}\right)$ and source term, respectively. As all problems with Lagrange multipliers, eq. (11) exhibits a saddle-point structure, making the system Jacobian A indefinite, possessing both positive and negative eigenvalues.

For 2D problems, the solver of choice is usually a sparse direct solver that can handle indefinite matrices, such as those implemented in UMFPACK, MUMPS, LU, SuperLU, and STRUMPACK. In 3D, preconditioned Krylov methods, such as MINRES or GMRES, can outperform parallel direct solvers. Other techniques to solve eq. (11) involve hybridization or static condensation techniques, such as generalized displacement methods (Fraeijs de Veubeke 1965; Arnold \& Brezzi 1985), domain decomposition approaches (Khattatov \& Yotov 2019), and elimination of the degrees of freedom of the stress around the vertices (e.g., Ambartsumyan et al. 2020). For example, Ambartsumyan et al. (2020) use a vertex quadrature rule for the stress bilinear form which allows for local eliminations of the stress in the case of $D G_{0}$ for rotation, resulting in a cell-centered displacement-rotation system, or eliminating both stress and rotation in the case of $D G_{1}$ which leads to a displacement-only cell-centered system. Several preconditioners are available to efficiently solve eq. (11) using iterative methods (e.g., Klawonn \& Starke 2004; Wildey \& Xue 2013; Bærland et al. 2017; Rees \& Wathen 2020). However some of the implementation is not trivial. For simplicity in this work, we used an efficient direct solver to avoid nuances in the implementation of such complex methods.

\subsection{Advantages and Disadvantages of Mixed FE Methods}

The classic approach to construct a FE discretization for the elasticity equation is represented by the pure displacement formulation method. Since the compliance material tensor $\boldsymbol{A}$ is invertible, the stress $\sigma$ can be eliminated by substituting the constitutive relationship into the conservation of linear 
momentum. After taking the dot product of the governing equations with a test function $v$ and setting the integral over $\Omega$ equal to zero, the weak form in this pure elastic displacement formulation reads

$\int_{\Omega} \boldsymbol{\sigma}(\boldsymbol{u}): \boldsymbol{\varepsilon}(v) d \boldsymbol{x}=\int_{\Omega} \boldsymbol{f} v d \boldsymbol{x}+\int_{\Gamma_{N}} \boldsymbol{t} v d s \quad \forall v \in \Upsilon$,

where $\Upsilon=\left\{v \in H_{0}^{1}(\Omega, \mathbb{V}): v=0\right.$ on $\left.\Gamma_{N}\right\}$ is the space of square integrable vector fields on $\Omega$, with square integrable derivatives which vanish on the Neumann boundary $\Gamma_{N}$. In general, the vector function space $\Upsilon$ is approximated by $\Upsilon_{h}=C G_{k}\left(\mathcal{T}_{h}, \mathbb{V}\right)$, which are the $k$-th order continuous piecewise polynomials belonging to the Lagrange elements. Note that in this case the displacement boundary condition is essential while the traction condition becomes natural.

A FE approach based on this pure displacement formulation is standard and found in many textbooks (e.g., Ciarlet 2002). However, such formulation is not preferable for more complex models in viscoelasticity (e.g., Rognes \& Winther 2010), poroelasticity (e.g., Bærland et al. 2017), plasticity (e.g., Johnson 1977) and Stokes problems (e.g., Stenberg 1984), where the stress-strain relation is not local and the stress variable $\boldsymbol{\sigma}$ cannot be eliminated (Arnold et al. 2007).

Another advantage of this mixed FE approach is that the stress is computed with one order higher accuracy than the pure displacement formulation (see Section 3.3). For pure displacement formulations, instead, the stress variable must be obtained a posteriori by differentiation leading to a loss of accuracy (Arnold 1990). The mixed formulation also results in conservation of momentum at the element level, not just at the global level. It is also well-known that the standard displacement discretization is not robust in the incompressible and nearly incompressible case, i.e. as $\lambda \rightarrow \infty$ (Arnold

et al. 1984b). While the elastic compliance tensor $\boldsymbol{A}$ is bounded as Poisson's ratio $\nu \rightarrow \frac{1}{2}$, its inverse blows up. For the elastic mixed method, instead, the compliance $\boldsymbol{A}$ tends to a limiting value (Arnold 1990).

There are also some disadvantages to these mixed approaches in comparison with displacement methods. While displacement methods typically lead to positive definite algebraic systems, we saw that the discretized system for the mixed method is indefinite, which for large scale problems requires specialized preconditioners in combination with iterative solvers (Section 3.1.1). Lastly, because both stress, displacement and rotation are calculated simultaneously, the discrete mixed system generally involves more degrees of freedom than displacement approaches (see Section 3.3.1).

\subsection{Benchmarks and Performance}

We now compare the elastic solution between the mixed finite-element approach (MF) and the pure displacement formulation (DF). We compute the corresponding convergence rate and analyze their performances. Then, always within the same FENICS framework, we compare the fault implementa- 


\section{S. Puel et al.}

tion described in Section 3 with the split node technique (Melosh \& Raefsky 1981) in the case of an in-plane crack mode II. We also compare both results with analytic solutions (Pollard \& Segall 1987).

The simulations and the convergence tests are performed using a single core of a laptop (eightcore Intel $\mathrm{I} 9-9880 \mathrm{H}$ machine running at $2.4 \mathrm{GHz}$ with $32 \mathrm{~GB}$ of RAM). We use the sparse LU factorization provided by Multifrontal Massively Parallel Solver (MUMPS) to solve our systems of equations (Amestoy et al. 2001, 2019). For simplicity and because of the 2D nature of our tests, we choose to use this robust and accurate direct solver rather than Krylov iterative solvers to fairly compare the performance of both formulations without preconditioning the systems.

\subsubsection{Verification}

To verify the accuracy of the mixed FE approach and compare it to the standard pure displacement formulation, we create an exact solution by applying the method of manufactured solutions (Roache 2002; Oberkampf \& Roy 2010). Considering a 2D connected domain $\Omega \subset \mathbb{R}^{2}$ with boundaries $\partial \Omega=$ $\Gamma_{D}$, the boundary value problem of the linear elasticity in the pure displacement formulation, with vanishing Dirichlet boundaries, reads

$$
\begin{cases}\nabla \cdot \boldsymbol{\sigma}=-\boldsymbol{f} & \text { in } \Omega, \\ \boldsymbol{\sigma}=2 \mu \boldsymbol{\varepsilon}+\lambda \operatorname{tr}(\boldsymbol{\varepsilon}) \boldsymbol{I} & \text { in } \Omega, \\ \boldsymbol{\varepsilon}=\frac{1}{2}\left(\nabla \boldsymbol{u}+(\nabla \boldsymbol{u})^{T}\right) & \text { in } \Omega, \\ \boldsymbol{u}=0 & \text { on } \Gamma_{D} .\end{cases}
$$

The forcing vector $f$ can be calculated such that the exact solution is given by

$$
\begin{aligned}
& \boldsymbol{u}_{\mathrm{ex}}=\left(\begin{array}{c}
0 \\
\sin (2 \pi x) \sin (2 \pi y)
\end{array}\right), \\
& \boldsymbol{\sigma}_{\mathrm{ex}}=\left(\begin{array}{cc}
\lambda 2 \pi \sin (2 \pi x) \cos (2 \pi y) & \mu 2 \pi \cos (2 \pi x) \sin (2 \pi y) \\
\mu 2 \pi \cos (2 \pi x) \sin (2 \pi y) & (2 \mu+\lambda) 2 \pi \sin (2 \pi x) \cos (2 \pi y)
\end{array}\right),
\end{aligned}
$$

where $\boldsymbol{u}_{\mathrm{ex}}$ is the exact solution for displacement, and the stress $\boldsymbol{\sigma}_{\mathrm{ex}}$ is recovered by substituting the exact displacement into eq. (13). Appendix A provides a derivation of the source term $f$ and stress $\sigma_{\text {ex }}$ expressions.

Now that we have the exact solution of our variables, we can verify and compare the accuracy between our mixed method and the pure displacement approach. In order to do so, we consider a unit square $\Omega:=[0,1] \times[0,1]$ with vanishing Dirichlet boundary conditions. We build the triangular mesh directly in FENICS using the built-in mesh function (Fig. S1). The body force $f$ is then determined using Lamé coefficients $\mu=1.0$ and $\lambda=2.0$. To fairly compare the two approaches, we choose to keep the same order of accuracy for the displacement variable. For linear elements, we discretize 
$\boldsymbol{u}$ with first order continuous polynomials for the pure displacement formulation, and $k=2$ for the mixed method. In the latter, the displacement field is discretized with linear discontinuous piecewise elements ( $D G_{1}$, Fig. 2). Fig. 3 shows the comparison of the displacement and stress magnitudes between the difference of the pure displacement approach and the mixed method with respect to the exact solution (eq. 14).

While the displacement is comparable between the two different methods (same color scale), the stress is not. As analyzed by many studies (e.g., Arnold et al. 2007; Cockburn et al. 2010; Am-
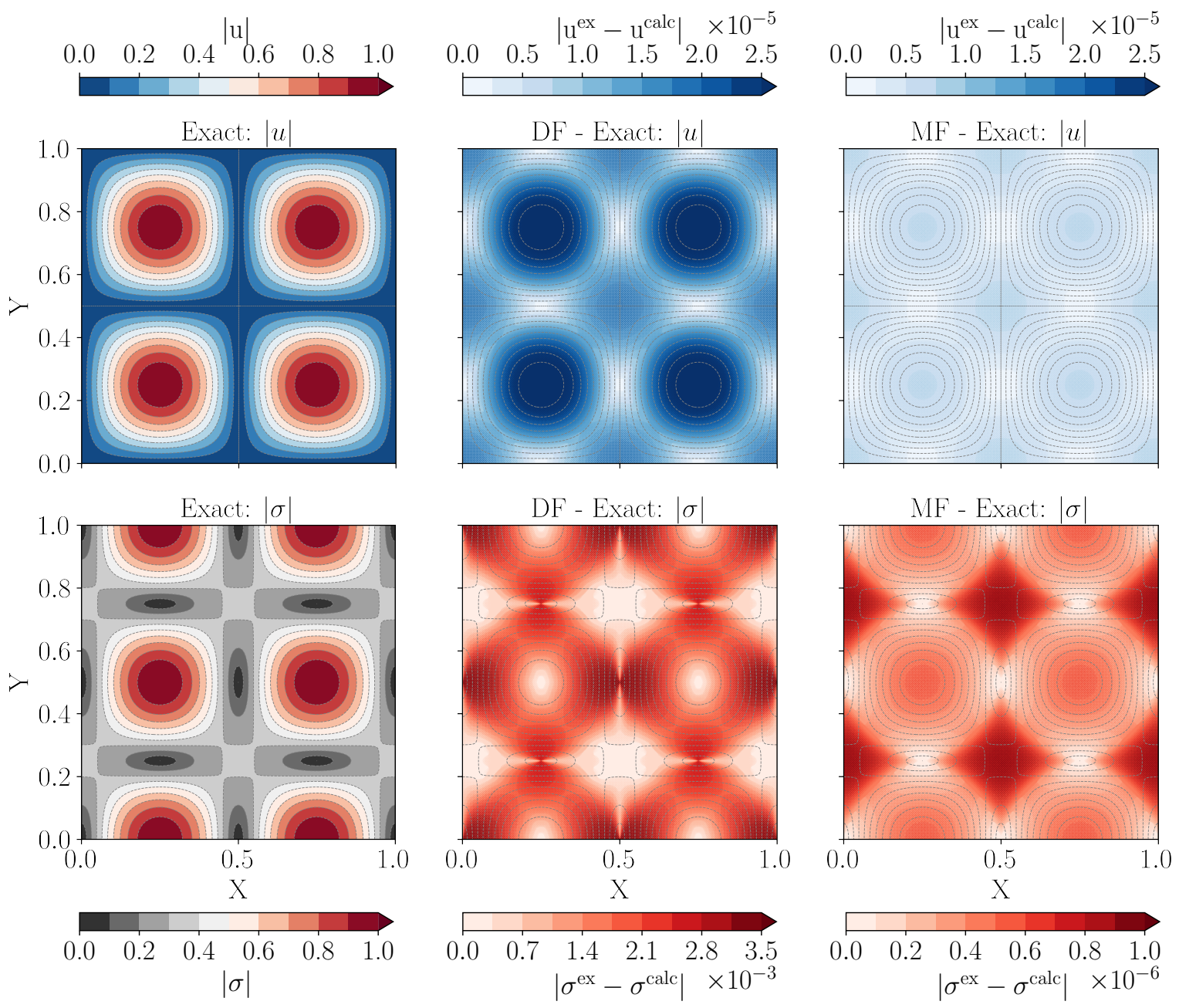

Figure 3. Comparison of the displacement and stress magnitudes between the pure displacement formulation (DF), the mixed method (MF), and the exact solution. Top left to right: displacement magnitude of the exact solution, and the absolute difference between DF and MF with respect to the exact solution, respectively. Bottom left to right: same as above, but for the magnitude of the stress field. Note that the color scale for the displacement difference is the same for DF and MF, while for the stress it differs by about three orders of magnitude. Linear elements for displacement are used in all computations, and cell size $h=1 / 512$. The stress magnitude results are normalized by the maximum exact values. 
bartsumyan et al. 2020), the mixed finite-element exhibits one order higher accuracy than the pure displacement formulation. Moreover, the error pattern differs between the two formulations for the magnitude of displacement and stress. While in DF the main displacement difference is concentrated at the four peaks (top center in Fig. 3), in the mixed method the error is slightly lower and more evenly distributed (top right). For the stress, the error distribution between DF and MF is quite different. The error for the pure displacement formulation is three orders of magnitude larger and is mainly concentrated in the slopes around the peaks (bottom center), while for the mixed method it is mostly focused at the corners of each quadrant (bottom right).

Given the exact solution and mesh size $h$, we can also compute the convergence rates of the two different elastic methods. For polynomials of order $k$, we expect the error to be $\mathcal{O}\left(h^{k+1}\right)$ and $\mathcal{O}\left(h^{k}\right)$ in the $L_{2}$ and $H_{1}$ norm, respectively. Fig. 4 shows the convergence rates for linear and quadratic elements for the displacement field. The theoretical rates in (a), (b), (e), and (f) are denoted with dashed lines. All rates are in agreement with the theoretical expectations, as indicated in (a), (b), (e), and (f). The mixed method possesses the same order of accuracy for both stress and displacement variables in their corresponding norms for both linear and quadratic elements (Arnold et al. 2007; Cockburn et al. 2010;
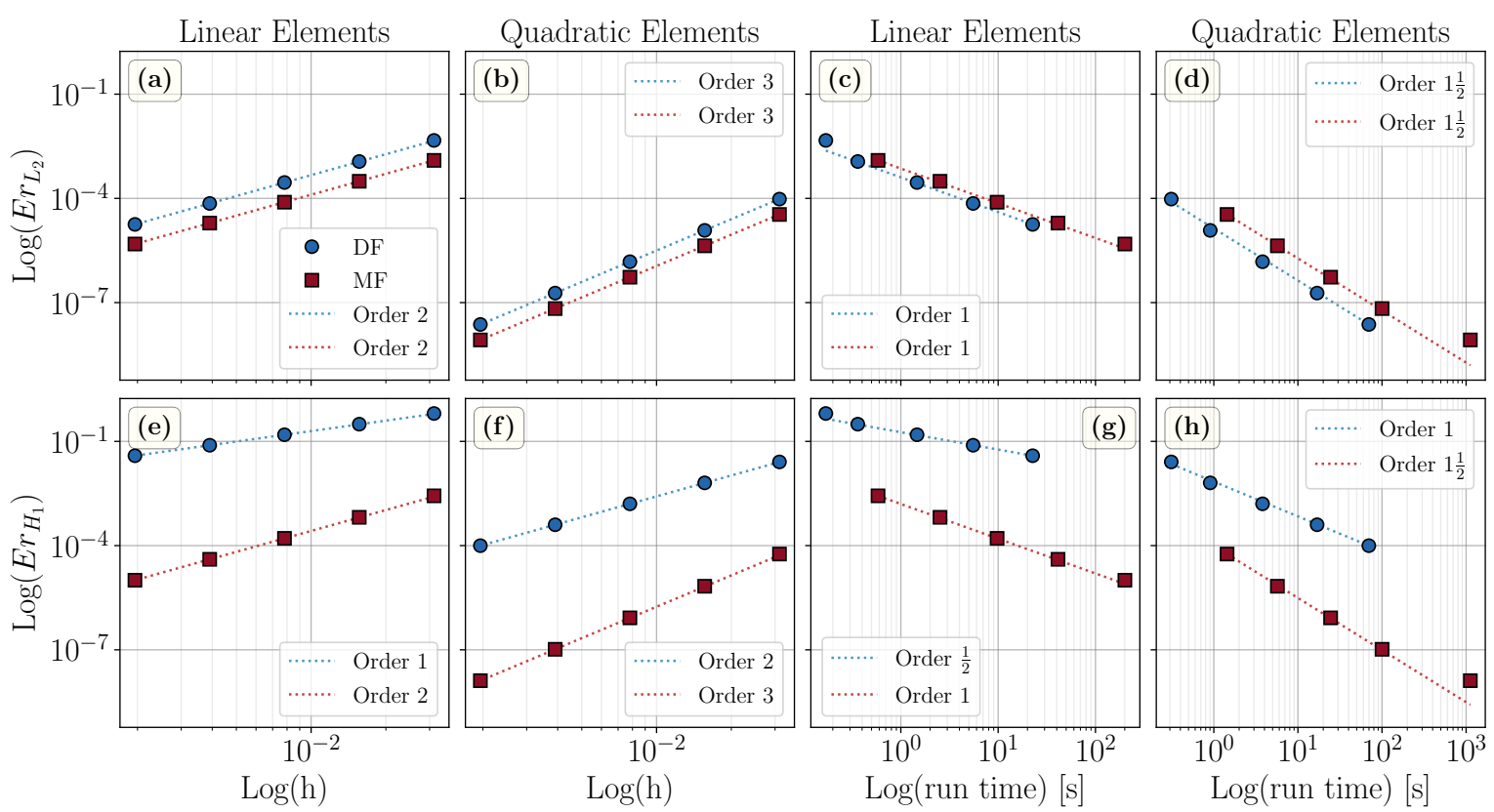

Figure 4. Convergence rate and performance comparisons between the pure displacement formulation (DF, blue dots) and the mixed method (MF, red squares). (a) and (b) show the log-log plots of the $L_{2}$ norm error as a function of mesh size $h$ for the displacement with linear and quadratic elements, respectively. (e) and (f), same as (a) and (b), but for the for the stress ( $H_{1}$ norm). Dashed lines in (a), (b), (e), and (f) denote the theoretical convergence rate. (c), (d), (g), and (h) show the corresponding error as a function of the computational time. In this case, dashed lines indicate the best fit. 
Ambartsumyan et al. 2020). Although the order of convergence for displacement is the same, the mixed approach is slightly more accurate than the standard elastic method for the same discretization.

Fig. 4 (c), (d), (g), and (h) show the total computational time vs. the error of the displacement (top) and stress (bottom) for linear and quadratic elements, respectively. Dashed lines indicate linear trends, whose rates are shown in the corresponding legend. For a target fixed accuracy in the displacement unknown the run times of DF and MF are comparable, the former being slightly faster than the latter. This may be due to to the greater number of degrees of freedom (DOFs) that the mixed system has (stress, displacement and rotation) than DF. Fig. S2 shows the total number of DOFs and computational time as a function of the mesh refinement. We can see that the number of DOFs for the mixed method are greater than those for the pure displacement formulation, with a ratio MF/DF of about 4.8 and 2.9 for linear and quadratic elements, respectively. This difference leads the computational time for MF to be about 6.1 and 5.8 times higher than DF.

However, for a fixed target accuracy in the stress variables, the run time of MF is several order of magnitude faster than DF, as seen in Fig. 4, panels (g) and (h). This demonstrates that the proposed MF formulation is preferable when accuracy in the stress variables is needed. For the very fine mesh and quadratic elements, the run time for the mixed method seems to slightly deviate from the trend. This may be due to loss of performance of MUMPS in solving very large problems (about $24 M$ of total DOFs).

\subsubsection{Comparison with analytical solution for a mode II crack}

To test the implementation of a fault discontinuity, we consider the case of an in-plane shear crack, mode II (e.g., Lawn \& Wilshaw 1975). Analytic expressions for the displacement and stress fields are in the form of (e.g., Pollard \& Segall 1987; Segall 2010; Scholz 2019)

$u_{i}=K_{I I} \frac{2(1-\nu)}{\mu} \sqrt{\frac{\boldsymbol{r}}{2 \pi}} f_{i}(\boldsymbol{\theta}) \quad$ and $\quad \sigma_{i j}=K_{I I} \sqrt{\frac{1}{2 \pi \boldsymbol{r}}} f_{i j}(\boldsymbol{\theta})$,

where $\mu$ and $\nu$ indicate the shear modulus and Poisson's ratio, respectively, $\boldsymbol{r}$ is the distance from the crack tip into the crack, and $\boldsymbol{\theta}$ is the angle measured from the crack plane. $K_{I I}$ is the stress intensity factor for mode II and depends on the geometry and magnitudes of the applied loads. Both $K_{I I}$ and the functions $f_{i}(\boldsymbol{\theta})$ and $f_{i j}(\boldsymbol{\theta})$ can be found in standard references (e.g., Lawn \& Wilshaw 1975; Tada et al. 1973). Appendix B provides the analytic expressions of the displacement and stress fields for a crack mode II used in this study and reported in Pollard \& Segall (1987).

Besides with the analytic solution, we compare the mixed method results with the pure displacement approach, in which the relative motion along crack walls is modeled by using the split node 
technique. For the implementation of the split node method within the FENICS framework, we follow the strategy first suggested by Melosh \& Raefsky (1981).

For the comparison between the different approaches, we consider a domain $\Omega:=[0,4] \times[0,4]$ with a crack of unity length located at the center of the domain (Fig. S3). We use the FENICS builtin function to build the mesh, and apply the analytic displacement $\left(\boldsymbol{u}=\boldsymbol{u}_{\text {an }}\right)$ at the boundaries (eq. B.2). For the elastic properties, a shear modulus of $\mu=1$ and Poisson's ratio of $\nu=0.25$ are used throughout the computations. We apply a unity stress drop, which leads to an elliptical solution for the slip (Appendix B) (Pollard \& Segall 1987; Scholz 2019). We prescribe a left-lateral movement, and perform the simulations using linear elements.

Fig. 5 shows the comparison of the displacement magnitude and the mean normal stress between the analytic, the pure displacement method with the split node technique, and the mixed approach. To make the absolute difference of DF and MF with respect to the analytic solution meaningful (second and third columns), we normalize the displacement magnitude by the maximum analytic slip (top), and the normal stress by its maximum analytic value (bottom). Both the mixed method and the pure displacement formulation with the split node technique are in good agreement with the analytic solution, with errors less than $\sim 0.05 \%$ for both displacement and stress. MF gives better results than DF. For the stress, this is expected since the mixed method has an order of accuracy higher than the standard displacement approach (Arnold et al. 2007; Cockburn et al. 2010; Ambartsumyan et al. 2020). The main differences are focused on the crack tips. In the pure displacement method with the split node technique, the systematic error is likely related to the stress singularities at the crack tips since the elastic solution is computed at the vertices. This problem can be overcome by the mixed method because the computation of the displacement solution occurs within each cell (see Fig. 2). We note a small difference at the crack tips, but this discrepancy is below $0.02 \%$ for both variables. Similar results are also visible in Figs S4 and S5, where we compare all the components of the displacement and stress field.

Lastly, we compute the convergence rates for the MF with the fault implementation described at the beginning of the section and DF with the split node technique. In order to avoid the systematic discrepancies around the crack tips (see Fig. 5), we remove a $0.5 \times 0.5$ block around the discontinuity, which corresponds to one cell in the coarser mesh case. We refine the mesh, and compute the error $L_{2}$ and $H_{1}$ norm for the displacement and stress, respectively, as we did for the manufactured solution case. Fig. 6 shows the comparison of the convergence rate between the mixed method (red) and the pure displacement formulation with the split node technique (blue), using linear elements. Empty and full symbols indicate the integration of the error over the entire domain and excluding the $0.5 \times 0.5$ block around the crack, respectively. 

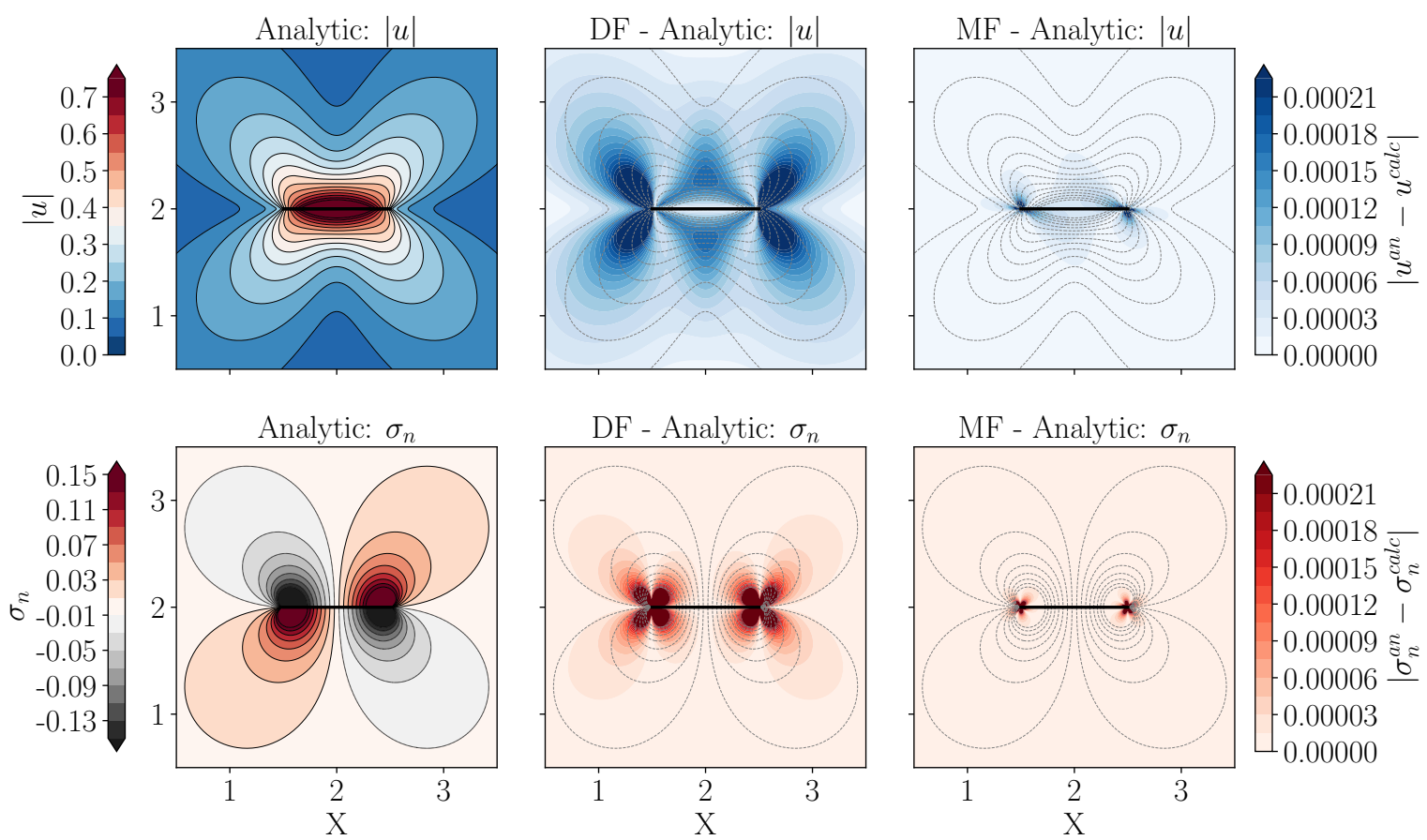

Figure 5. Comparison of the displacement magnitude and mean normal stress between the pure displacement formulation with the split node technique (DF), the mixed method with the fault implementation described in Section 3 (MF), and the analytic solution for a shear crack, mode II (Pollard \& Segall 1987). Top left to right: displacement magnitude of the analytic solution, and the absolute difference between DF and MF with respect to the analytic solution, respectively. Bottom left to right: same as above, but for the mean normal stress. Linear elements for displacement are used in all computations, and cell size $h=1 / 128$. The displacement magnitude and the mean normal stress results are normalized by the maximum analytic slip and by the maximum analytic value, respectively.

Considering the entire domain, the convergence rates do not agree with the theoretical ones (dotted lines), likely due to the systematic errors around the crack tips (Fig. 5). In particular, for the displacement the error decreases as the mesh refines at the rate of about 0.45 for both DF and MF. For the stress, instead, the convergence rate is negative. This opposite trend may be related to the presence of the stress singularities at the crack tips. Refining the mesh, the singularity may be better approximated by the stress solution increasing the corresponding error. Removing the small area around the discontinuity in the error calculation, the convergence rates significantly improve, approaching the theoretical ones. For the displacement, while the MF follows the theoretical curve, the convergence for the DF with the split node technique is slightly lower than the theoretical one ( $1 \frac{1}{2}$ instead of 2 ). This small discrepancy may be related to the effect of the displacement around the crack tips beyond the small region around the discontinuity that we excluded in the error calculation (cf. Fig. 5). 

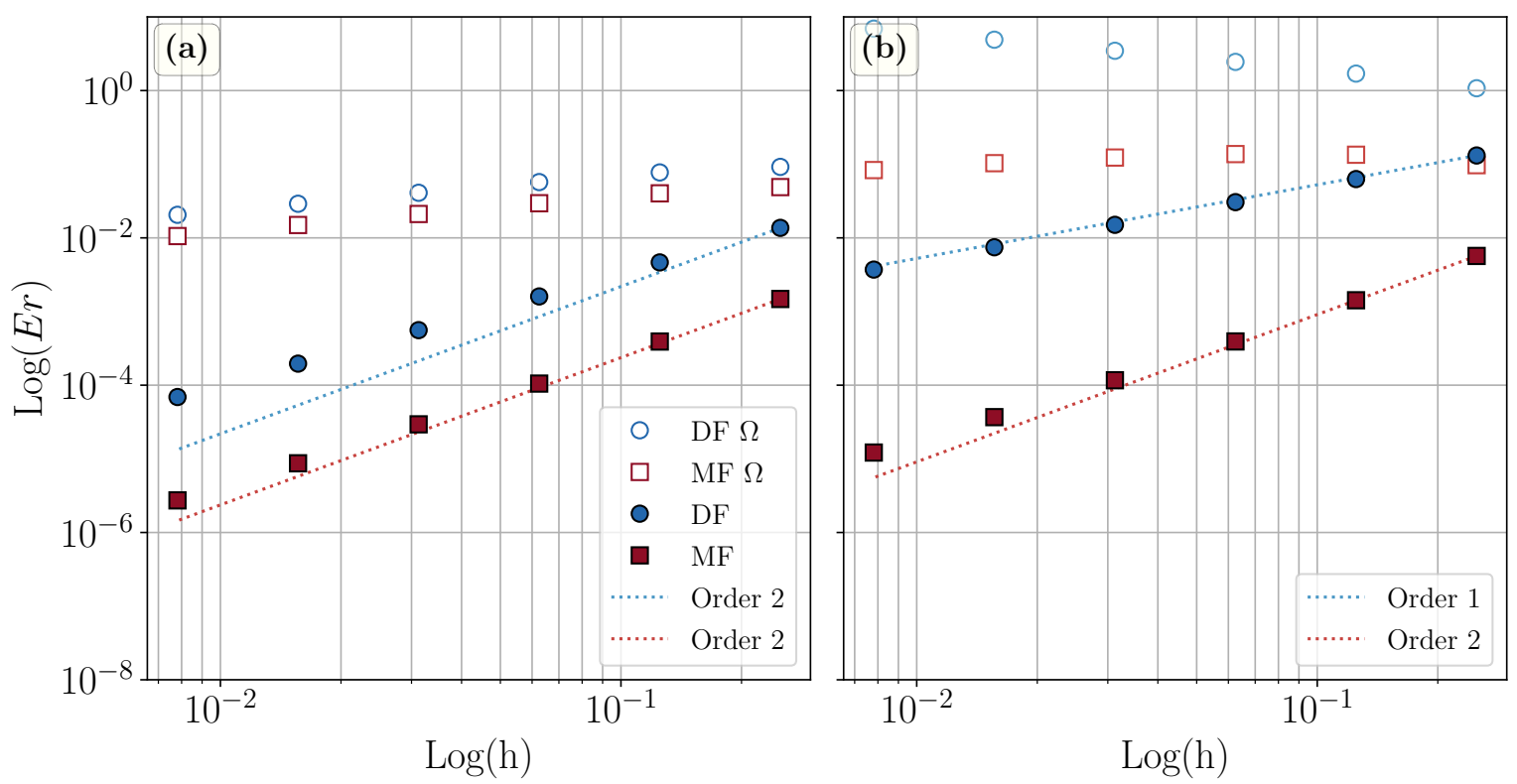

Figure 6. Convergence rate comparison between the mixed method (MF, red squares) and the pure displacement formulation with the split node technique (DF, blue dots), using linear elements. Empty and full symbols indicate the integration of the error over the entire domain and excluding the $0.5 \times 0.5$ block around the crack, respectively. (a) shows the log-log plot of $L_{2}$ norm error for the displacement as a function of the mesh size $h$, while (b) the $H_{1}$ norm error for the stress. Dashed lines denote the theoretical convergence rates.

\section{THE INVERSE PROBLEM: COSEISMIC SLIP DISTRIBUTION}

We now pose the inverse problem: given a set of surface displacement data recorded with different observations (e.g., GPS, InSAR, etc.), we seek to find the fault slip responsible for such deformation. One of the strengths of the proposed FENICS-HIPPYLIB framework is that the forward and inverse problems can be performed within the same continuum formulation, in a flexible, transparent and easily extensible way. Although the HIPPYLIB library contains many algorithms to solve the inverse problem in a Bayesian fashion to better quantify model uncertainties, here we show an application of this new framework to a classic earthquake problem, the linear deterministic inversion for the coseismic slip distribution. However, we stress that the transition between the two inverse formulations is straightforward (e.g., Isaac et al. 2015) and all the underlying algorithms are contained in HIPPYLIB (Villa et al. 2021).

The reason for initially addressing the linear slip inversion problem is twofold: first, we want to exploit our treatment of the fault discontinuity within the mixed FE approach, since the slip appears directly in the right hand-side of the constitutive law (eq. 8) after integration by parts. This fault implementation presents a straightforward path to derive the gradient corresponding to the slip field without discretizing the fault a priori (which avoids differentiating through numerical artifacts) and computing 
the Green's functions. Second, we can compare our results with the standard approach of inverting the matrix of Green's functions (Okada 1992) to solve the linear inverse problem. Although HIPPYLIB automatically computes gradient and Hessian information by applying symbolic differentiation to the variational form of the forward problem (eq. 8), in the next section we briefly introduce the concept behind in the context of our coseismic slip inversion.

\subsection{The Adjoint Method for the Coseismic Slip Problem}

Given some discrete, noisy observations $\mathbf{d} \in \mathbb{R}^{n_{o b s}}$, the goal of the inverse problem is to infer the unknown model parameter field $m \in \mathcal{M}$ that best reproduces the observations. Mathematically, this relationship can be written as

$\mathbf{d}=\mathcal{F}(\boldsymbol{m})+\boldsymbol{\eta}$,

where $\mathcal{F}: \mathcal{M} \rightarrow \mathbb{R}^{n_{\text {obs }}}$ is the parameter-to-observable map, describing the process that predicts the data for a given parameter $\boldsymbol{m}$, and $\boldsymbol{m}$ is the slip field $s$ in eq. (8) for the linear coseismic slip inversion. $\boldsymbol{\eta}$ indicates additive noise due to uncertainties in the data and model errors (Tarantola 2005). In HIPPYLIB, the noise is modeled as a Gaussian distribution $\boldsymbol{\eta} \sim \mathcal{N}\left(0, \Gamma_{\text {noise }}\right)$ centered at 0 with covariance $\Gamma_{\text {noise }}$. The mapping $\mathcal{F}$ is given by a linear or non-linear observation operator $\mathcal{B}(\varphi): \mathcal{U} \rightarrow$ $\mathbb{R}^{n_{\text {obs }}}$ that extracts the observations from the states $\varphi \in \mathcal{U}$, where $\varphi$ depend on $\boldsymbol{m}$ via the solution of the forward problem or state equation. In the case of the coseismic slip inversion, the states $\varphi$ correspond to displacement, stress, and rotation, respectively, and the mapping $\mathcal{F}(\boldsymbol{m})$ is linear since the slip appears linearly in eq. (8). This mapping can be discretized as $\mathbf{F m}=\mathbf{B} \mathbf{A}^{-1} \mathbf{M m}$, where $\mathbf{F}$ is the discretized parameter-to-observable map, and $\mathbf{m}$ is the slip vector. $\mathbf{B}$ is the discretized observation operator $\mathcal{B}$, which evaluates the displacement $\boldsymbol{u}$ at the observation locations. $\mathbf{A}$ and $\mathbf{M}=\left(M_{F},-M_{\Omega}, 0\right)^{T}$ are the mixed elasticity and mass matrices of eq. (11), respectively. In our case, the source term $f$ is zero, hence $M_{\Omega}$ vanishes.

The main challenge of solving eq. (17) is that, in the general case of $\mathcal{F}$ governed by PDEs with infinite-dimensional parameters, the inverse problem is ill-posed, i.e., the solution is not unique and highly sensitive to errors in the data (Hadamard 1923; Tikhonov \& Arsenin 1977; Engl et al. 1996). To overcome this issue, we usually regularize the problem by including additional information on the solution, such as smoothness (Vogel 2002).

In general, we can formulate the linear inverse problem as follows: given a set of finite-dimensional noisy measurements $\mathbf{d} \in \mathbb{R}^{n_{o b s}}$, we seek to find the model parameter $\boldsymbol{m}$ which can predict the data 
within the noise tolerance. This translates into solving the following optimization problem:

$\min _{\boldsymbol{m} \in \mathcal{M}} \mathcal{J}(\boldsymbol{m}):=\frac{1}{2}\|\mathcal{F}(\boldsymbol{m})-\mathbf{d}\|_{\Gamma_{\text {noise }}^{-1}}^{2}+\mathcal{R}(\boldsymbol{m})$.

Here, the cost functional $\mathcal{J}(\boldsymbol{m})$ consists of two terms. The first is the misfit between the observations $\mathbf{d}$ and those predicted by the mapping $\mathcal{F}(\boldsymbol{m})$, weighted by the inverse of the data noise covariance $\Gamma_{\text {noise }}^{-1}$. The second term $\mathcal{R}(\boldsymbol{m})$ is the regularization, which penalizes oscillatory components of the model parameter $\boldsymbol{m}$ by imposing some sort of regularity, such as smoothness. In the case of the coseismic slip, we may use some type of Tikhonov regularization (Phillips 1962; Tikhonov 1963) that penalizes, for example, the gradient or the second derivative of the model parameter, allowing the solution to vary smoothly.

We propose to infer the fault slip by solving the optimization problem of eq. (18). In general, to efficiently solve this linear least-squares problem, first (gradient) and second derivative (Hessian) information of $\mathcal{J}(\boldsymbol{m})$ are needed. In our case, only the gradient depends on the model parameter, since the forward problem is linear, while the Hessian is independent of $\boldsymbol{m}$. The gradient expression can be derived by using the Lagrangian formalism (Tröltzsch 2010).

For the linear elastic problem, the Lagrangian functional for the infinite-dimensional gradient $\mathcal{L}^{\mathcal{G}}$, in variational form, reads

$$
\begin{aligned}
\mathcal{L}^{\mathcal{G}}(\boldsymbol{\sigma}, \boldsymbol{u}, \boldsymbol{r}, \boldsymbol{m}, \tau, \omega, \xi) & =\frac{1}{2} \int_{\Omega}(\mathcal{B} \boldsymbol{u}-\mathbf{d})^{T} \Gamma_{\text {noise }}^{-1}(\mathcal{B} \boldsymbol{u}-\mathbf{d}) d \boldsymbol{x} \\
& +\frac{1}{2} \int_{\Omega}\left[\gamma\left[\nabla\left(\boldsymbol{m}-\boldsymbol{m}_{0}\right)\right]^{2}+\delta\left(\boldsymbol{m}-\boldsymbol{m}_{0}\right)^{2}\right] d \boldsymbol{x} \\
& +\int_{\Omega} \boldsymbol{A} \boldsymbol{\sigma}: \tau d \boldsymbol{x}+\int_{\Omega} \boldsymbol{u} \cdot(\nabla \cdot \tau) d \boldsymbol{x}+\int_{\Omega} \operatorname{as}(\tau) \cdot \boldsymbol{r} d \boldsymbol{x} \\
& -\int_{\Gamma_{D}} \boldsymbol{u}_{0} \cdot(\tau \mathbf{n}) d S-\int_{\Gamma_{F}} \boldsymbol{m} \cdot\left[T\left(\mathbf{n}^{+}\right)\left(\tau^{+} \mathbf{n}^{+}\right)\right] d S \\
& +\int_{\Omega}(\nabla \cdot \boldsymbol{\sigma}) \cdot \omega d \boldsymbol{x}+\int_{\Omega} \boldsymbol{f} \cdot \omega d \boldsymbol{x}+\int_{\Omega} \operatorname{as}(\boldsymbol{\sigma}) \cdot \xi d \boldsymbol{x},
\end{aligned}
$$

where the first term is the data misfit, and the second is a linear combination of a $H^{1}$ and $L^{2}$-type Tikhonov regularization that penalizes the $H^{1}(\Omega)$ and $L^{2}(\Omega)$ norm of $\left(\boldsymbol{m}-\boldsymbol{m}_{0}\right)$, respectively. $\boldsymbol{m}_{0}$ is a reference model parameter, while $\gamma$ and $\delta$ represent smoothing weights. The other terms form the residual of the forward PDE model (eq. 8), where $\tau, \omega$ and $\xi$ represent auxiliary variables, called the adjoint variables, for the stress, displacement and rotation, respectively. We have replaced the slip $s$ of eq. (8) with the unknown parameter field $\boldsymbol{m}$ we seek to infer from the data. Note that, although the slip is defined on $\Gamma_{F}$, we need to integrate the model parameter on the entire domain $\Omega$. This is due to a current limitation in FENICS to manage function spaces defined on different meshes (e.g., domain and fault).

By setting to zero the variation of the Lagrangian $\mathcal{L}^{\mathcal{G}}$ with respect to the adjoint variables, one ob- 
tains the variational form of the forward problem (eq. 8) to find the displacement, stress, and rotation, respectively. Similarly, by setting to zero the variation of the Lagrangian $\mathcal{L}^{\mathcal{G}}$ with respect to the state variables one obtains the weak form of the so-called adjoint problem:

$$
\begin{aligned}
\left(\frac{\partial \mathcal{L}^{\mathcal{G}}}{\partial \boldsymbol{\sigma}}, \tilde{\sigma}\right)+\left(\frac{\partial \mathcal{L}^{\mathcal{G}}}{\partial \boldsymbol{u}}, \tilde{u}\right)+\left(\frac{\partial \mathcal{L}^{\mathcal{G}}}{\partial \boldsymbol{r}}, \tilde{r}\right) & =\int_{\Omega} \boldsymbol{A} \tilde{\sigma}: \tau d \boldsymbol{x}+\int_{\Omega}(\nabla \cdot \tilde{\sigma}) \cdot \omega d \boldsymbol{x}+\int_{\Omega} \operatorname{as}(\tilde{\sigma}) \cdot \xi d \boldsymbol{x} \\
& +\int_{\Omega} \tilde{u} \cdot(\nabla \cdot \tau) d \boldsymbol{x}+\int_{\Omega} \mathcal{B}^{*} \Gamma_{\text {noise }}^{-1}(\mathcal{B} \boldsymbol{u}-\mathbf{d}) \cdot \tilde{u} d \boldsymbol{x} \\
& +\int_{\Omega} \operatorname{as}(\tau) \cdot \tilde{r} d \boldsymbol{x} \quad \forall \tilde{\sigma} \in \Sigma ; \forall \tilde{u} \in W ; \forall \tilde{r} \in \Xi
\end{aligned}
$$

where $\tilde{\sigma}, \tilde{u}$, and $\tilde{r}$ are test functions, and $\mathcal{B}^{*}: \mathbb{R}^{n_{\text {obs }}} \rightarrow \mathcal{U}$ is the inverse mapping of $\mathcal{B}$ that maps the discrete observations back to the infinite-dimensional space of the states $\mathcal{U}$. We can solve the adjoint equation above to find the adjoint variables $\tau, \omega$ and $\xi$. In contrast with the strong form of the forward problem in eq. (1), the strong form of the adjoint problem reads

$$
\begin{cases}\boldsymbol{A} \tau-\nabla \omega+\operatorname{as}^{*}(\xi)=0 & \text { in } \Omega, \\ \nabla \cdot \tau=-\mathcal{B}^{*} \Gamma_{\text {noise }}^{-1}(\mathcal{B} \boldsymbol{u}-\mathbf{d}) & \text { in } \Omega, \\ \operatorname{as}(\tau)=0 & \text { in } \Omega, \\ \omega=0 & \text { on } \Gamma_{D} \\ \tau \cdot \mathbf{n}=0 & \text { on } \Gamma_{N}\end{cases}
$$

where $\operatorname{as}^{*}(\xi)$ is defined such that: $\int_{\Omega} \operatorname{as}(\tilde{\sigma}) \cdot \xi d \boldsymbol{x}=\int_{\Omega} \tilde{\sigma}: \operatorname{as}^{*}(\xi) d \boldsymbol{x}$, and in 2D takes the form of

$$
\operatorname{as}^{*}(\xi)=\left(\begin{array}{cc}
0 & -\xi \\
\xi & 0
\end{array}\right) .
$$

Lastly, we can derive the gradient of the cost functional by taking the variation of the Lagrangian with respect to the model parameter. The gradient of $\mathcal{J}(\boldsymbol{m})$ in an arbitrary direction $\tilde{m} \in \mathcal{M}$, evaluated at an arbitrary point $m^{*}$ in the parameter space $\mathcal{M}$, is the Gâteaux derivative of $\mathcal{L}^{\mathcal{G}}$ with respect to $\boldsymbol{m}$ :

$$
\begin{aligned}
\left(\mathcal{G}\left(m^{*}\right), \tilde{m}\right) & =\gamma \int_{\Omega} \nabla\left(m^{*}-m_{0}\right) \cdot \nabla \tilde{m} d \boldsymbol{x}+\delta \int_{\Omega}\left(m^{*}-m_{0}\right) \cdot \tilde{m} d \boldsymbol{x} \\
& -\int_{\Gamma_{F}} \tilde{m} \cdot\left[T\left(\mathbf{n}^{+}\right)\left(\tau^{+} \mathbf{n}^{+}\right)\right] d S \quad \forall \tilde{m} \in \mathcal{M},
\end{aligned}
$$

where we can see that the gradient is linear in $m^{*}$ since $\tau^{+}$depends linearly on $\boldsymbol{u}$ via the solution of the adjoint problem (eq. 21), and $\boldsymbol{u}$ depends linearly on $m^{*}$ via solution of the (weak) forward mixed elasticity problem (eq. 8). The terms in the gradient expression (eq. 23) that depend linearly on $m^{*}$ define the Hessian operator, whose action on an arbitrary $m^{*}$ entails solution of one forward (eq. 8) and one adjoint (eq. 20) mixed elasticity problem. Integrating by parts the regularization term, the 
strong form of the gradient expression then reads

$\mathcal{G}(\boldsymbol{m})= \begin{cases}-\gamma \Delta\left(\boldsymbol{m}-\boldsymbol{m}_{0}\right)+\delta\left(\boldsymbol{m}-\boldsymbol{m}_{0}\right) & \text { in } \Omega, \\ \gamma \llbracket \nabla\left(\boldsymbol{m}-\boldsymbol{m}_{0}\right) \cdot \mathbf{n} \rrbracket-\left[T\left(\mathbf{n}_{\Gamma_{F}}\right)\left(\tau \mathbf{n}_{\Gamma_{F}}\right)\right] & \text { on } \Gamma_{F}, \\ \gamma \nabla\left(\boldsymbol{m}-\boldsymbol{m}_{0}\right) \cdot \mathbf{n} & \text { on } \partial \Omega,\end{cases}$

where $\mathbf{n}_{\Gamma_{F}}=\mathbf{n}^{+}=-\mathbf{n}^{-}$. In HIPPYLIB, we can either explicitly input the expression for the gradient as in eq. (23), or else let HIPPYLIB derive this expression using FENICS's symbolic capability for taking variations of weak forms.

To efficiently solve the linear inverse problem (eq. 18), we use a preconditioned conjugate gradient (CG) algorithm to solve the first order necessary condition,

$\left(\mathcal{G}\left(m^{*}\right), \tilde{m}\right)=0 \quad \forall \tilde{m} \in \mathcal{M}$

for $m^{*}$. At each CG iteration, a Hessian action must be computed, which as stated above entails solution of a pair of forward/adjoint mixed elasticity problems. Preconditioning the system by the inverse of the regularization operator $\mathcal{R}$ transforms the Hessian into the sum of a compact operator (its eigenvalues accumulate at zero) and an identity operator (Villa et al. 2021), for which CG is known to converge rapidly and in a number of iterations that does not depend on the parameter dimension or the data dimension (Ghattas \& Willcox 2021). Therefore, the overall cost of solving the inverse problem, measured in forward/adjoint problem solutions, does not depend on the parameter or data dimensions, and instead depends only on the intrinsic information contained in the data about the model (e.g. Bui-Thanh et al. 2012, 2013; Isaac et al. 2015; Ghattas \& Willcox 2021).

\subsection{Comparison with Standard Coseismic Slip Inversion Approach}

For a linear inverse problem, we can rewrite eq. (17) in its discrete form, omitting the noise term, as

$\mathbf{d}=\mathbf{G m}$

where the data kernel $\mathbf{G}$ relates the model parameter vector $\mathbf{m}=\left[m_{1}, m_{2}, \ldots, m_{M}\right]^{T}$ to the finitedimensional observations $\mathbf{d}=\left[d_{1}, d_{2}, \ldots, d_{N}\right]^{T}$. For the coseismic slip problem, the fault surface is generally discretized a priori into rectangular patches, and every column of the $N \times M$ matrix $\mathbf{G}$ contains the surface displacements at the observation locations computed by imposing unity slip for each fault patch, using the elastic Green's functions within an elastic half-space (Okada 1992).

The inverse problem defined by eq. (26) is usually ill-posed due either to having more unknown parameters than data (lack of uniqueness) or to having very small singular values (lack of stability) (Tarantola 2005). Therefore, we need to add some a priori information on $\mathbf{m}$ to constrain the solution 
(Jackson 1979). This prior knowledge can be encapsulated in a regularization term, analogous to $\mathcal{R}(\boldsymbol{m})$ in eq. (18).

We can solve the linear inverse problem of eq. (26) by using, for example, a weighted damped least-squares approach (Menke 2018):

$\mathbf{m}=\mathbf{G}^{-g} \mathbf{d} \quad$ where $\quad \mathbf{G}^{-g}=\left(\mathbf{G}^{T} \mathbf{G}+\beta \mathbf{L}^{T} \mathbf{L}\right)^{-1} \mathbf{G}^{T}$,

where $\mathbf{L}$ is a linear operator identified with the Tikhonov regularization $\mathcal{R}(\boldsymbol{m})$ in eq. (18), and $\beta$ is a weighting parameter. In particular, if $\mathbf{L}$ is the identity, the regularization is called zeroth-order Tikhonov. If the operator is the gradient or the Laplacian, we refer to it as first and second-order Tikhonov regularization, respectively. Zeroth-order Tikhonov regularization is not commonly used; the majority of coseismic studies typically decide to penalize the gradient or the second derivative of the model parameters (e.g., Liu \& Archuleta 2004; Hsu et al. 2006, 2011; Liu et al. 2019; Wang et al. 2020). However, this standard approach encapsulates many limitations. This method requires a priori fault discretization, and the computational time to calculate the slip rises up as the number of subfault patches increases. Additionally, a realistic complex geometry of the fault and 3D heterogeneous media may be difficult to explore with this approach, mainly due to computational limitations.

\subsubsection{Comparison of the Inversion Results}

To compare the results of the coseismic slip inversion between the standard linear approach and the adjoint method via the FENICS-HIPPYLIB framework, we consider a 2D model with a curved fault and 20 observations uniformly-spaced at the surface (inset in Figs 7 and 8). For the adjoint linear inversion, we consider a rectangular computational domain of size $1100 \times 500 \mathrm{~km}$. The open-source software GMSH (Geuzaine \& Remacle 2009) is used to generate an unstructured mesh with 12,930 triangular cells. The mesh is finer in a region near the fault, mesh size $\sim 5 \mathrm{~km}$, and coarser near the vertical and bottom boundaries. The mixed elasticity problem is discretized using the $1^{\text {st }}$ order stable triplet of finite element spaces, resulting in 117,062 DOFs for the state variables (stress, displacement and rotation). Fig. 7 shows the discretized domain, where the fault discontinuity is divided in 22 uniformly-spaced segments of $5 \mathrm{~km}$ each. The same fault geometry is used to compute the data kernel $\mathbf{G}$ of Green's functions in eq. (26).

A homogeneous isotropic Earth's elastic structure is considered, but a fully heterogeneous medium can be easily implemented within our framework. Table 1 summarizes the parameters used in our computation. At the fault boundary, we prescribe a Gaussian slip centered at $20 \mathrm{~km}$ depth and standard deviation of $15 \mathrm{~km}$ resembling an earthquake nucleated at shallow depth on the subduction interface.

We apply zero displacement boundary conditions to the left, right and bottom boundaries, and 


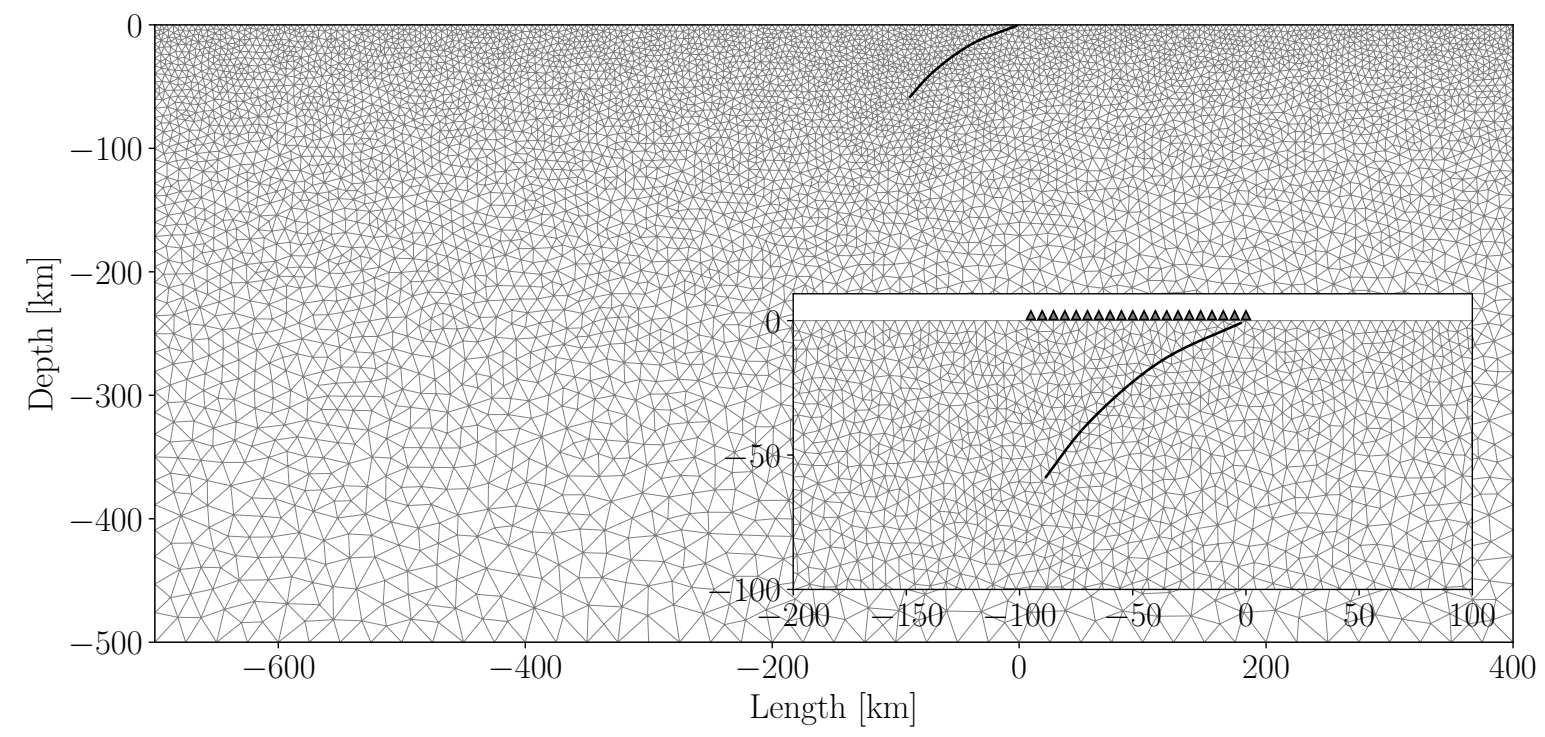

Figure 7. Unstructured triangular mesh used for the coseismic slip inversion. The black solid line indicates the curved fault. Inset: zoom in the area around the fault discontinuity. The grey triangles represent the locations of the 20 surface observations.

a free surface at the top of the model. Although the model extends for several fault lengths, it is not sufficient to fully remove the effects of the boundary conditions. This produces a slightly different displacement field between the numerical and the analytic solution moving away from the fault source. The standard solution would again involve increasing the domain size. However, this is not an issue in our case since we are interested in demonstrating the solution of the coseismic slip inversion with synthetic (simulated) data. As long as the boundary conditions of the forward model used to generate the synthetic data are consistent with those used for the inversion, the quality of the inverse solution is not affected by the choice of the boundary conditions.

For the classic slip inversion, we compute the data kernel G of Green's functions by Okada's routine "DC3D" (Okada 1992). In our FENICS-HIPPYLIB framework, we compute synthetic hori-

Table 1. Elastic properties and geometry parameters for the coseismic slip inversion.

\begin{tabular}{lclc}
\hline Elastic property & Value & Geometry parameter & Value \\
\hline Density $(\rho)$ & $2670 \mathrm{~kg} / \mathrm{m}^{3}$ & Domain length & $1100 \mathrm{~km}$ \\
Shear modulus $(\mu)$ & $34 \mathrm{GPa}$ & Domain depth & $500 \mathrm{~km}$ \\
Lamé parameter $(\lambda)$ & $30 \mathrm{GPa}$ & Fault length & $110 \mathrm{~km}$ \\
& & Number fault segments & 22 \\
& & Fault slip depth mean & $20 \mathrm{~km}$ \\
& & Fault slip depth std & $15 \mathrm{~km}$ \\
\hline
\end{tabular}


zontal and vertical surface deformations by solving the forward problem (eq. (8), Fig. 8) and extract the displacement values at the 20 observations using the observation operator $\mathcal{B}(\boldsymbol{u})$. Fig. 8 shows the horizontal and vertical displacement field given the prescribed Gaussian slip, and the locations of the surface observations. The imposed Gaussian slip produces the largest surface deformation close to the $4^{\text {th }}, 5^{\text {th }}$, and $6^{\text {th }}$ stations from the left of the intersection between the fault interface and the surface. Moreover, there is a sharp and resolved jump of the displacement field between the two sides of the fault. This confirms the power of the mixed FE approach to deal with fault discontinuities.

To perform the coseismic slip inversion, we apply $5 \%$ random noise to the surface observations and calculate the noise variance as the product of the relative noise level and the $L_{\infty}$ norm of observed surface deformation. Since the inverse problem is ill-posed, we regularize the system with a regularization term $\mathcal{R}(\boldsymbol{m})$ of the form of eq. (19). We penalize both the magnitude and the gradient of the model parameter field so that the preconditioner $\mathcal{R}(\boldsymbol{m})$ is invertible, as needed by the CG algorithm. Therefore, we need a small contribution of the mass matrix. In a Bayesian inference setting, the ratio $\sqrt{\gamma / \delta}$ also plays the role of the correlation length in the prior term. After polluting the synthetic hor-

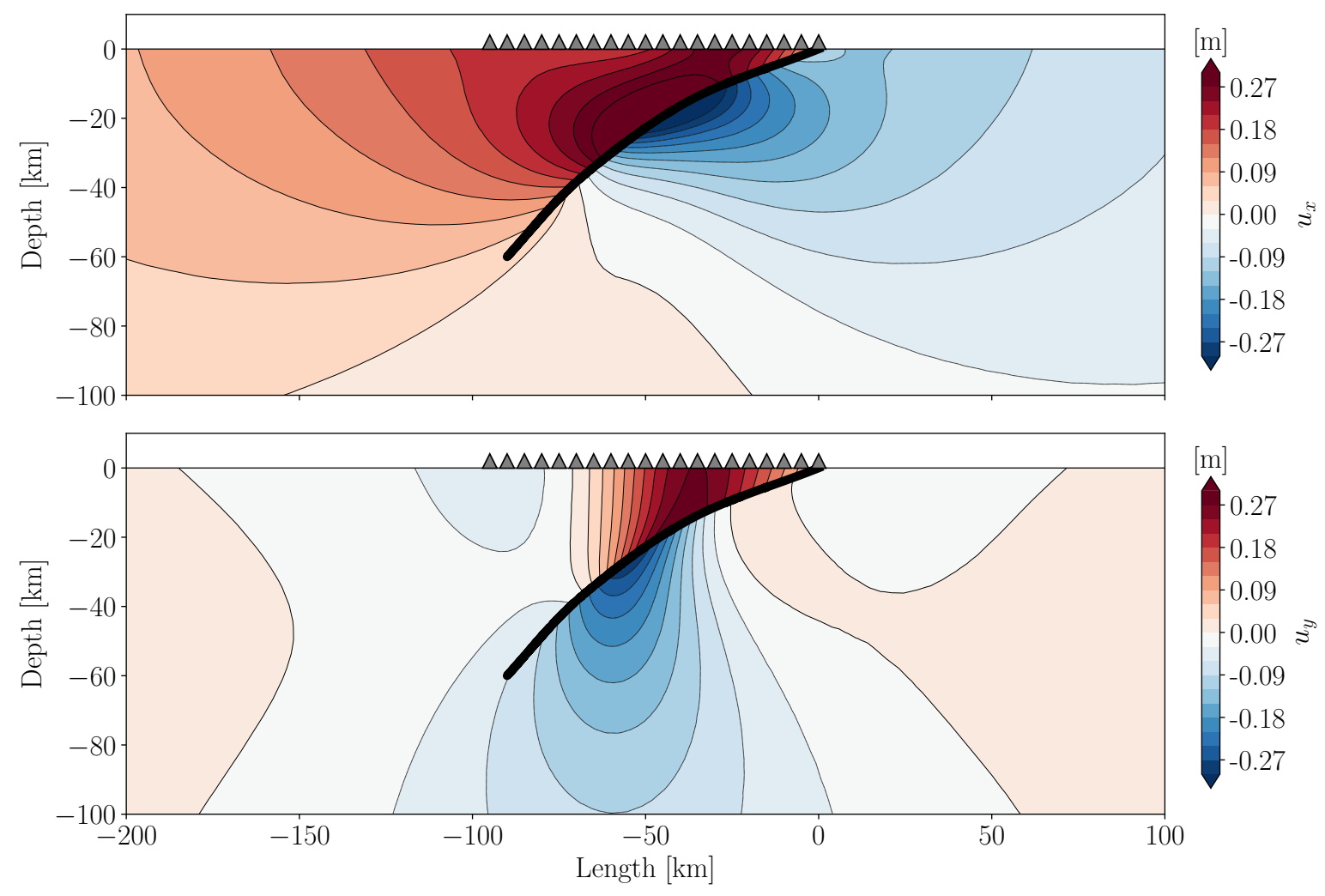

Figure 8. Horizontal ( $u_{x}$, top) and vertical ( $u_{y}$, bottom) displacement field given the prescribed Gaussian slip. Thick black solid line indicates the fault discontinuity. The grey triangles indicate the locations of the 20 surface observations. 
izontal and vertical surface data with the random noise, we fix the ratio $\gamma / \delta$ to be $10^{4}$, and perform an L-curve analysis (Miller 1970; Lawson \& Hanson 1995) to find the best value of $\gamma$. We obtain an "optimal" value of $\gamma=60$ (Fig. S6a).

To fairly compare the inversion results between the adjoint method and the standard approach using linear inverse theory, we need to discretize the fault slip in the same way. The data kernel $\mathbf{G}$ of Green's functions is calculated by imposing unity slip in each fault patch, and each patch is characterized by constant slip. To reproduce the same condition in the FENICS-HIPPYLIB framework, we use Crouzeix-Raviart $\left(C R_{1}\right)$ elements to discretize the fault slip, where the DOF is located at the mid-point of each fault segment. It is easy enough to discretize the slip using linear elements (i.e., using $C G_{1}$ elements), but in this case we would have one more DOF for slip than the standard linear method.

The result of our inversion is shown in Fig. 9. With this configuration of noise and regularization, the CG method converges in 24 iterations. At each CG iteration a pair of forward (eq. 8) and adjoint (eq. 20) problems are solved. In the same figure, we also plot the results of the coseismic slip inversion using linear inverse theory. We only plot the results for the first and second-order Tikhonov regularization, where the values of the corresponding weights are inferred from the L-curve criterion (Figs S6b and S6c). The slip distribution inferred using the adjoint method (Fig. 9a) approaches the true solution (black line). The result is comparable with the standard linear approach using Green's functions (b and c). The non-perfect match is due to the limited amount of surface data (only 20 observations) and noise level (5\%). Fig. S7 replicates the same inversion with lower noise (1\%) in which case the inverted slip very closely matches the true slip.

\subsubsection{Spectrum Decomposition}

Due to the linear nature of the coseismic slip inverse problem, the second derivative of the objective function (eq. 18), the so-called Hessian $\mathcal{H}$, is independent of the model parameter field $\boldsymbol{m}$ and data d. The analysis of the Hessian spectrum is useful to: 1) characterize the degree of ill-posedness of the inverse problem; 2) understand the redundancy of the data, and 3) determine which data contain more information about the infinite-dimensional field $\boldsymbol{m}$.

After discretization, the Hessian is generally a large, dense matrix; therefore, an explicit construction of $\mathbf{H}$ for large-scale problems is typically intractable since its dimension is equal to the dimension of $\boldsymbol{m}$. Each column of the Hessian requires the solution of a pair of linearized forward/adjoint PDEs. To make operations with the Hessian tractable, it is well-known that eigenvalues of the Hessian typically collapse rapidly to zero, since the data only contain limited information about the infinitedimensional parameter field. Hence, we can build a low-rank approximation of the data misfit compo- 


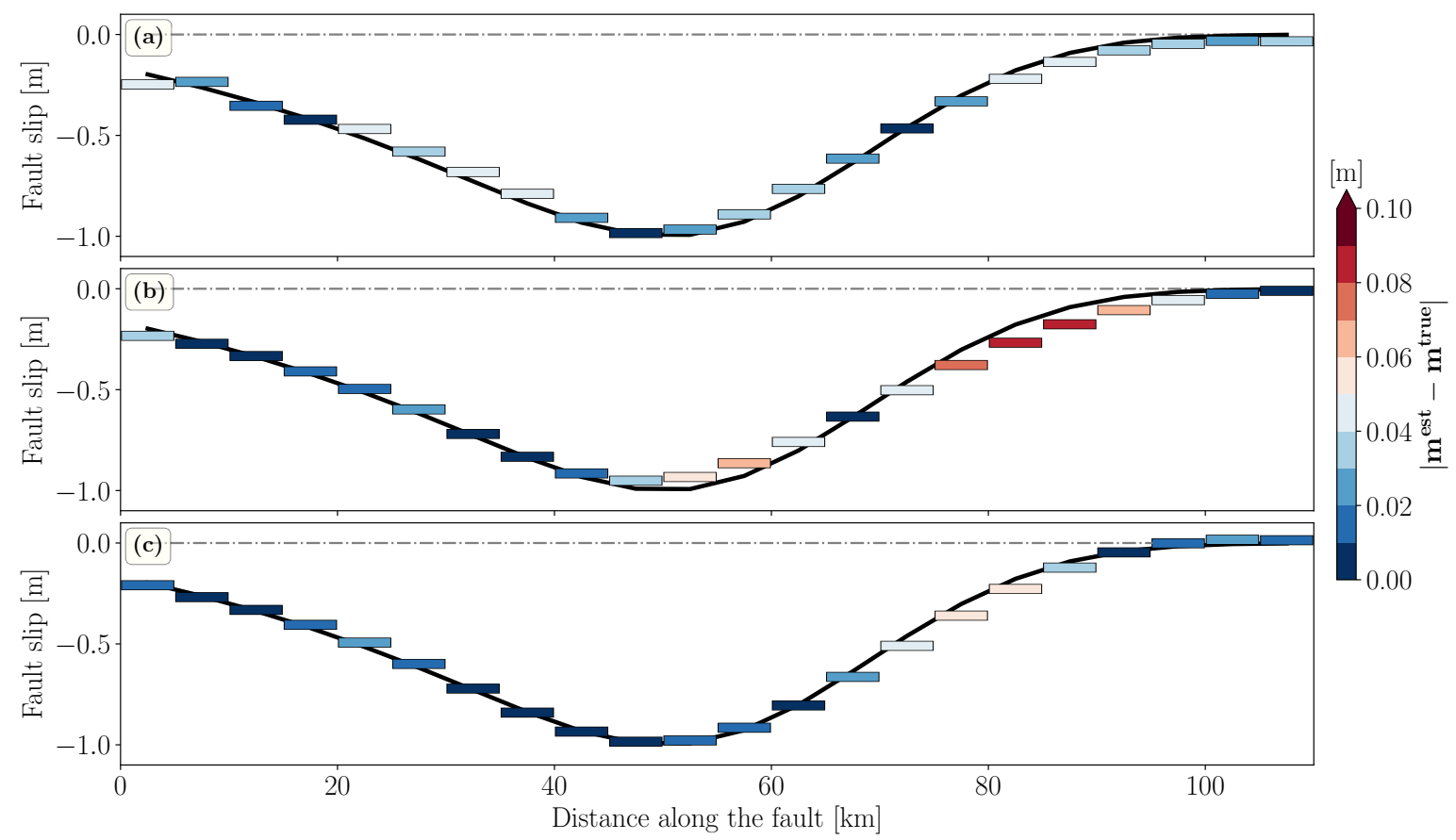

Figure 9. Reconstructed slip from the coseismic slip linear inversion along the fault. Zero distance (left) indicates the surface. (a) Fault slip estimated using the new framework and the adjoint method. The slip is constant within each subfault patch (22 total), and it is represented by horizontal colored segments. Each slip segment is colored by the absolute error with respect to its true value. (b)-(c) show the same inversion results as (a) but using the standard linear approach with first-order (b) and second-order (c) Tikhonov regularization, respectively. The data are polluted with $5 \%$ random Gaussian noise with zero mean and covariance $\Gamma_{\text {noise }}$.

nent of the Hessian, $\mathbf{H}^{\text {misfit }}$. The low-rank properties of $\mathbf{H}^{\text {misfit }}$ have been analytically demonstrated for many complex forward PDE problems (e.g. Bui-Thanh \& Ghattas 2013; Hesse \& Stadler 2014; Petra et al. 2014; Worthen et al. 2014; Isaac et al. 2015; Chen et al. 2019; Alghamdi et al. 2020)

The discretized Hessian can be decomposed into two components: the Hessian of the data misfit and the Hessian of the regularization,

$\mathbf{H}=\mathbf{H}^{\text {misift }}+\mathbf{H}^{\text {reg }}$,

where $\mathbf{H}^{\text {misfit }}$ has eigenvalues that decay to zero, reflecting ill-posedness. This property invites a low rank approximation of $\mathbf{H}^{\text {misfit }}$, which in HIPPYLIB we compute via a randomized eigensolver (Halko et al. 2011) to solve the following symmetric eigenproblem (Villa et al. 2021):

$\mathbf{H}^{\text {misift }} \mathbf{v}_{i}=\lambda_{i} \mathbf{v}_{i} \quad \lambda_{1} \geq \lambda_{1} \geq \ldots \geq \lambda_{n}$

where $\mathbf{v}_{i}$ is the eigenvector associated with the eigenvalue $\lambda_{i}$. The Hessian of the data misfit, $\mathbf{H}^{\text {misfit }}$, is a symmetric positive semi-definite matrix, and it can be easily related to the data kernel G. From 
eq. (27) we can see that the term in parenthesis of $\mathbf{G}^{-g}$ can be decomposed into a data misfit term and a regularization component. The data misfit Hessian can be discretized as $\mathbf{H}^{\text {misift }}=\frac{1}{\sigma_{\mathrm{d}}^{2}} \mathbf{F}^{T} \mathbf{F}=$ $\frac{1}{\sigma_{\mathrm{d}}^{2}} \mathbf{M} \mathbf{A}^{-1} \mathbf{B}^{T} \mathbf{B} \mathbf{A}^{-1} \mathbf{M}$, where $\sigma_{\mathrm{d}}^{2}$ is the data noise variance and we used the symmetry of $\mathbf{M}$ and $\mathbf{A}$. We note that $\mathbf{G} \sim \mathbf{F}$, in the sense that $\mathbf{G}$ corresponds to a different discretization technique, using Green's functions, of the same parameter to observable map. Hence, the data misfit Hessian $\mathbf{H}^{\text {misfit }}$ corresponds to $\mathbf{G}^{T} \mathbf{G}$ in the linear inverse theory.

Fig. 10 compares the spectrum between the data misfit Hessian and $\mathbf{G}^{T} \mathbf{G}$ for the coseismic slip inverse problem of Fig. 9. Both the eigenvalue decay (a) and the eigenvectors (b-c) are very similar. We will use the spectrum information of Fig. 10 to compare the resolution of the model parameters between the two approaches, and infer preliminary uncertainties of our coseismic slip inversion.

\subsubsection{Truncated SVD Solution, Model Resolution, and Uncertainty Analysis}

We can write the data kernel $\mathbf{G}$ using the singular-value decomposition (SVD) as

$\mathbf{G}=\mathbf{U} \boldsymbol{\Lambda} \mathbf{V}^{T}$

where $\mathbf{U}$ and $\mathbf{V}$ are $N \times N$ left and $M \times M$ right matrices of singular vectors, respectively, and $\boldsymbol{\Lambda}$ is an $N \times M$ diagonal matrix whose diagonal elements are called singular values. These singular values are non-negative and usually arranged in decreasing order. Some of them may be zero, and thus $\boldsymbol{\Lambda}$ can be partitioned into a $p \times p$ matrix $\boldsymbol{\Lambda}_{p}$ containing nonzero singular values and a matrix with zero entries. The subscript $p$ is an integer indicating how many singular values are positive (Menke 2018).
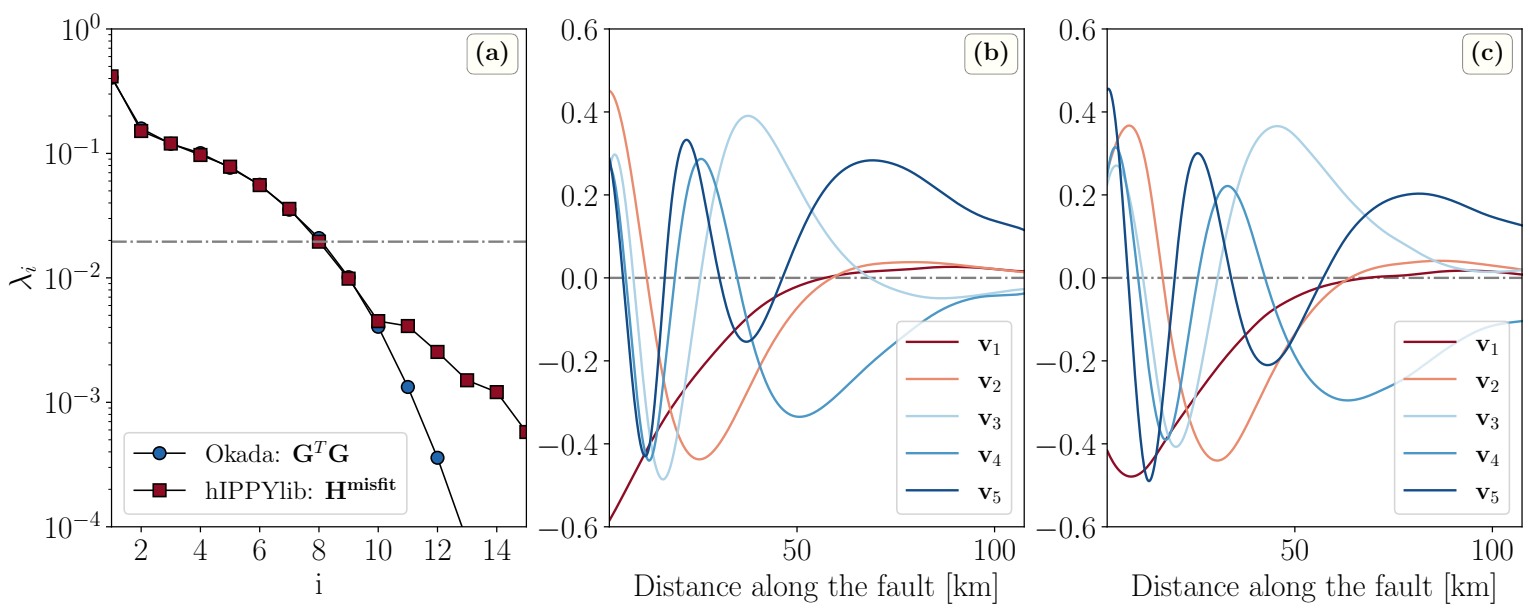

Figure 10. Comparison of spectra between the data misfit Hessian and the data kernel. (a) Eigenvalues decay for the coseismic slip problem. Blue dots and red squares indicate the eigenvalues $\lambda$ of $\mathbf{G}^{T} \mathbf{G}$ and $\mathbf{H}^{\text {misfit }}$, respectively. (b) First five eigenvectors of $\mathbf{G}^{T} \mathbf{G}$ (standard approach). (c) same as (b) but for the Hessian data misfit $\mathbf{H}^{\text {misfit }}$ using the adjoint method. 
Hence we can rewrite eq. (30) as $\mathbf{G}=\mathbf{U}_{p} \boldsymbol{\Lambda}_{p} \mathbf{V}_{p}^{T}$, where $\mathbf{U}_{p}$ and $\mathbf{V}_{p}$ consist of the first $p$ columns of $\mathrm{U}$ and $\mathbf{V}$, respectively.

The linear operator $\mathbf{G}$ is usually explicitly constructed, so its SVD decomposition is relatively straightforward. However, the computation of $\mathbf{U}, \boldsymbol{\Lambda}$, and $\mathbf{V}$ for the Hessian requires some care. Since the Hessian is typically a large, (formally) dense matrix for large-scale geophysical problems, unless the parameter dimension is modest, it is not possible to explicitly construct $\mathbf{H}$ and compute the SVD of the discretized parameter-to-observable map $\mathbf{F} \sim \mathbf{G}$. In order to address this issue and compute the SVD of $\mathbf{F}$ given the data misfit Hessian, we can readily build the columns of the right matrix of singular vectors $\mathbf{V}$ from the eigenvectors $\mathbf{v}_{i}$ of eq. (29). The diagonal matrix $\boldsymbol{\Lambda}$ can be easily calculated by taking the square roots of the eigenvalues $\lambda_{i}$ of eq. (29). Finally, the $N \times N$ left matrix of singular vectors $\mathbf{U}$ requires the knowledge of the discretized observation operator $\mathbf{B}$, the mixed elasticity matrix $\mathbf{A}$, and the mass matrix $\mathbf{M}$ :

$\mathbf{U}_{i}=\frac{\mathbf{B A}^{-1} \mathbf{M} \mathbf{V}_{i}}{\sqrt{\lambda_{i}}}$

From this SVD decomposition of the data kernel $\mathbf{G}$ and Hessian, the truncated SVD solution of the linear inverse problem (eq. 27) can be obtained by

$\mathbf{m}^{\mathrm{est}}=\mathbf{V}_{p} \boldsymbol{\Lambda}_{p}^{-1} \mathbf{U}_{p}^{T} \mathbf{d}$,

where the integer $p$ must be chosen such that the smallest eigenvalues are excluded from the calculation (Menke 2018). The grey dashed line in Fig. 10a indicates the choice of the value of $p$ for our coseismic slip inverse problem. We choose $p=8$ because it gives a good natural solution of the inverse problem from SVD (Fig. 11). Lower or higher values of $p$ do not improve the truncated SVD solutions. We may obtain better results by lowering the noise level in the data.

According to linear inverse theory, we can write the model resolution matrix of the natural generalized inverse, $\mathbf{R}$, as

$\mathbf{R}=\mathbf{G}^{-g} \mathbf{G}=\left\{\mathbf{V}_{p} \boldsymbol{\Lambda}_{p}^{-1} \mathbf{U}_{p}^{T}\right\}\left\{\mathbf{U}_{p} \boldsymbol{\Lambda}_{p} \mathbf{V}_{p}^{T}\right\}=\mathbf{V}_{p} \mathbf{V}_{p}^{T}$

The model resolution matrix characterizes whether each model parameter is uniquely determined (if $\mathbf{R}$ is the identity matrix). If $\mathbf{R}$ is not an identity matrix, the estimates of $\mathbf{m}$ are weighted averages of the true model parameters.

Fig. 12 shows the comparison of the model resolution matrix between the standard approach (a) and the adjoint method based on the mixed elasticity formulation (b) for our coseismic slip problem. The model resolution matrices look very similar. Both plots show high resolution near the top left, indicating that the shallow slip is well resolved, while the resolution becomes poor at depth. This is expected, since we have observations only at the surface (see Fig. 8). Note that $\mathbf{R}$ does not depend 


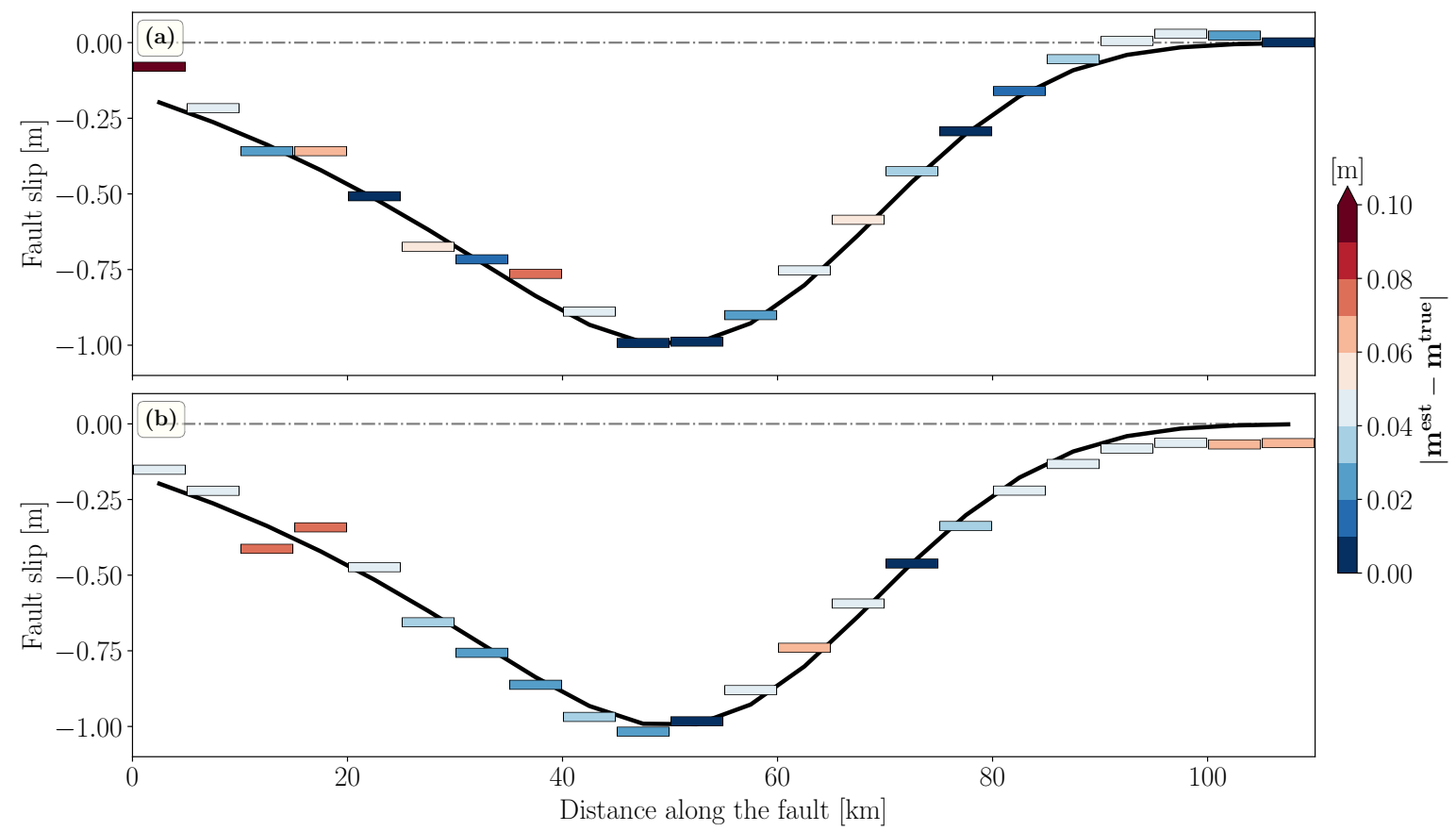

Figure 11. Reconstructed slip from the coseismic slip linear inversion using SVD decomposition of the Hessian misfit $\mathbf{H}^{\text {misfit }}$ (a) and the data kernel $\mathbf{G}$ (b). We choose $p=8$ to avoid to include the smallest eigenvalues (see Fig. 10a). The data are polluted with $5 \%$ random Gaussian noise with zero mean and covariance $\Gamma_{\text {noise }}$.

on the actual values of the data, but only on the fault geometry, observation location, and a priori information added to the problem (Menke 2018).

The data kernel and Hessian spectra can also provide information about the uncertainties in the model parameters. Given statistically uncorrelated observational errors with uniform variance $\sigma_{\mathrm{d}}^{2}$, it is possible to calculate how the error in the data propagates through the inversion process, leading to estimates of model parameters with covariance $\mathbf{C}_{\mathrm{m}}$. We can also rewrite this model covariance matrix using SVD. The pointwise variance of $\mathbf{m}$ is given by the diagonal elements of the model covariance matrix,

$$
\begin{aligned}
\mathbf{C}_{\mathrm{m}}=\mathbf{G}^{-g} \mathbf{C}_{\mathrm{d}} \mathbf{G}^{-g T} & =\sigma_{\mathrm{d}}^{2}\left\{\mathbf{V}_{p} \boldsymbol{\Lambda}_{p}^{-1} \mathbf{U}_{p}^{T}\right\}\left\{\mathbf{V}_{p} \boldsymbol{\Lambda}_{p}^{-1} \mathbf{U}_{p}^{T}\right\}^{T}=\sigma_{\mathrm{d}}^{2} \mathbf{V}_{p} \boldsymbol{\Lambda}_{p}^{-2} \mathbf{V}_{p}^{T}, \\
\sigma_{\mathrm{m}}^{2} & =\left[\mathbf{C}_{\mathrm{m}}\right]_{i i},
\end{aligned}
$$

where $\mathbf{C}_{\mathrm{d}}=\sigma_{\mathrm{d}}^{2} \mathbf{I}$ is the data covariance matrix. The diagonal matrix of singular values, $\boldsymbol{\Lambda}_{p}$, can be easily calculated by taking the square root of the eigenvalues $\lambda_{i}$ (eq. 29 and Fig. 10a). The model variances $\sigma_{\mathrm{m}}^{2}$ are typically used to infer the confidence bounds for the model parameters. Fig. 13 shows the results of the coseismic slip inversion shown in Fig. 9 within the $2 \sigma_{\mathrm{m}}^{2}=95 \%$ confidence intervals. As expected, resolution is highest at the shallow depths. This is also reflected by smaller confidence 

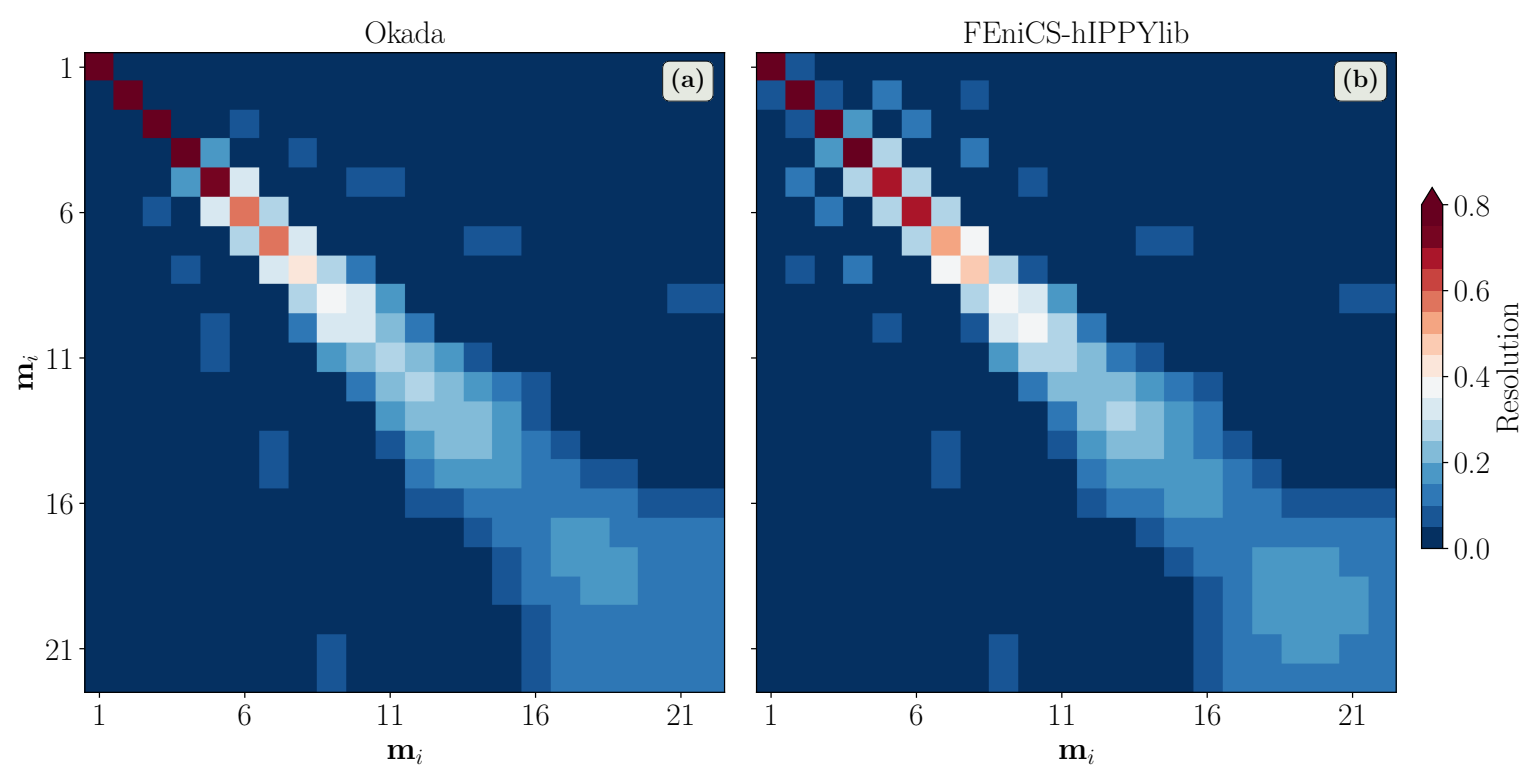

Figure 12. Comparison of the model resolution matrix between the standard linear inversion approach using the data kernel of Green's functions and the proposed adjoint method using the new framework. (a) Model resolution matrix calculated using SVD decomposition (eq. 33) of the data kernel $\mathbf{G}$ of Green's functions. (b) same as (a) but for the Hessian data misfit $\mathbf{H}^{\text {misfit }}$. The red colors indicate higher resolution near the surface, as expected.

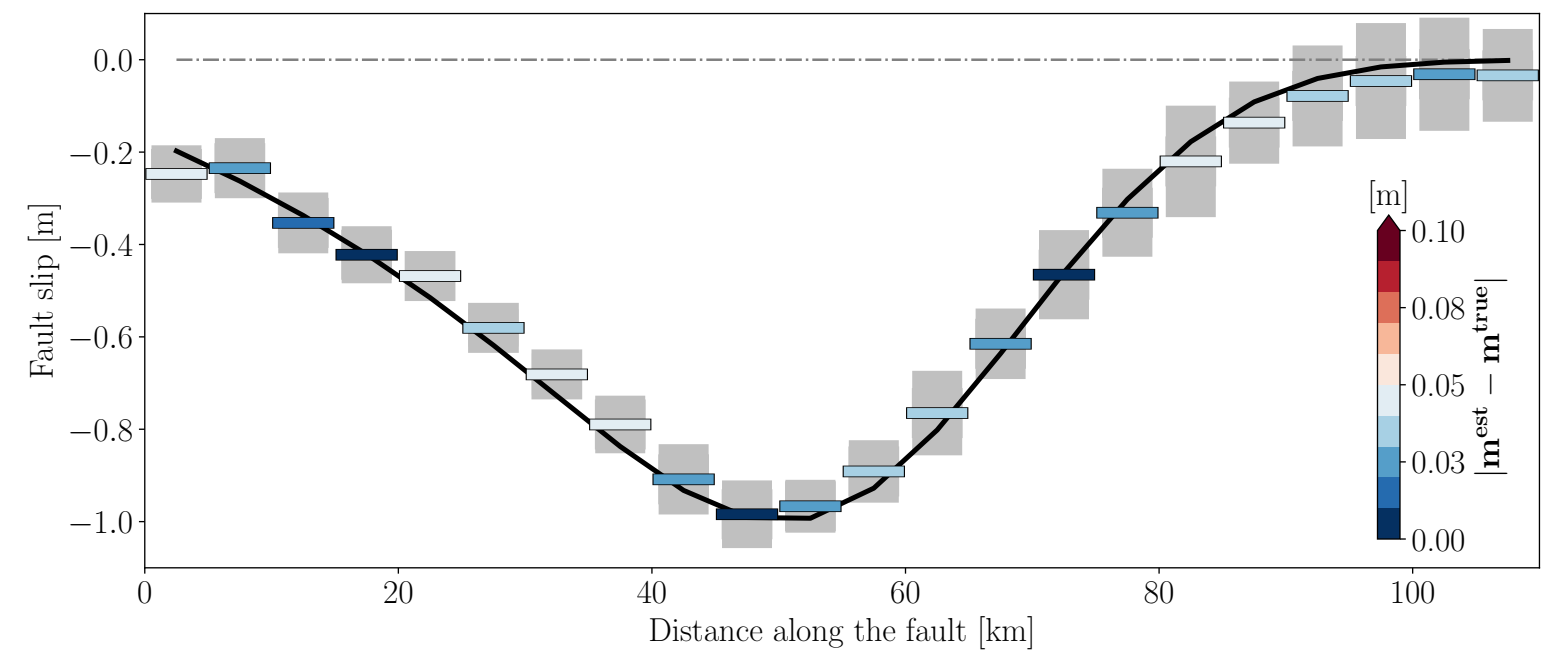

Figure 13. Inversion fault slip results using the new proposed framework. The figure is the same as Fig. 9a but with estimated model uncertainties. The grey boxes indicate the $2 \sigma_{\mathrm{m}}^{2}=95 \%$ confidence intervals calculated using SVD decomposition (eq. 34). 


\section{S. Puel et al.}

intervals in the slip solution close to the surface than at depths, where the coseismic slip may not be predicted with high accuracy due to the limited surface observations (20) and high data noise (5\%).

\section{CONCLUSIONS}

We developed a new, open-source FE modeling framework able to solve forward and inverse earthquake problems within the same computational architecture. This FENICS-HIPPYLIB framework provides the user with all the flexibility and transparency of the two advanced numerical libraries. Although suited for many multi-physics problems, we have focused on the coseismic slip problem and provided a new and rigorous fault implementation in a mixed finite element formulation at the continuum level. This allows the formulation of the coseismic slip inverse problem to expose the unknown slip field at the infinite dimensional level, thereby bypassing numerical artifacts and assumptions of the fault discretization (such as piecewise-constant slip and piecewise-linear fault geometry). It avoids the underlying assumptions of the Green's function approach, including the assumption of homogeneity, and permits the gradient and Hessian to be readily derived at the infinite dimensional level. This allows the discretization to be chosen in a manner that is appropriate for other needs such as sufficient accuracy and smoothness. The new formulation provides an attractive framework for slip inversion in a heterogeneous medium, for joint slip-medium inversion, and for inversion of the fault geometry.

The mixed elastic formulation exhibits a stress convergence rate that is one order higher than that of the pure displacement method, both theoretically and observed in numerical convergence tests. Moreover, the new fault implementation is more accurate near crack tips than the split node technique implemented within the standard displacement elastic formulation. While the new method is slower than the displacement approach for the same resolution (due to a larger number of degrees of freedom), it is far faster for the same stress accuracy-we observed several orders of magnitude speedup in runtime. The larger computational cost for the same mesh size may be remedied by the use of existing efficient preconditioners with iterative solvers, domain decomposition methods, and local elimination techniques, or a combination thereof. When applying the new framework to a classic earthquake problem, inversion for coseismic slip, results are comparable to the standard Green's function approach. From a spectral decomposition of the data misfit Hessian, we can estimate preliminary model uncertainties, and we document correspondence between the Hessian and data kernel spectra.

These promising results for our general forward and inverse framework indicate great utility for a number of more sophisticated earthquake problems. In a forthcoming paper, we will extend this modeling framework to perform non-linear and Bayesian inference inversions for more rigorous uncertainty quantification and inversions for heterogeneous material parameters, which are impossible with standard Green's function approaches. The flexibility of our new framework should allow for the 
rigorous integration of multi-physics and heterogeneous datasets, providing a new tool to help answer fundamental questions in earthquake science.

\section{ACKNOWLEDGMENTS}

We thank Dr. Ilona Ambartsumyan for the fruitful discussions about the mixed finite-element elastic formulation. S.P., T.W.B, and D.L. were supported by NSF EAR-2045292, 19214743, and 1927216. E.K., U.V., and O.G. were supported by NSF ACI-1550593 and DOE ASCR DE-SC0019303.

\section{DATA AVAILABILITY}

The fully documented Jupyter notebooks to reproduce the results are included as additional supporting files for the reviewers, and they will be available for the readers in an online repository via GitHub once the manuscript will be accepted. The FENICS and HIPPYLIB libraries can be download at https://fenicsproject.org and https://hippylib.github.io, respectively. The unstructured mesh for the FE simulation of the coseismic slip inversion was built using the opensource software GMSH (Geuzaine \& Remacle 2009) and the files are provided as supporting files.

\section{REFERENCES}

Aagaard, B. T., Knepley, M. G., \& Williams, C. A., 2013. A domain decomposition approach to implementing fault slip in finite-element models of quasi-static and dynamic crustal deformation, J. geophys. Res., 118(6), 3059-3079.

Aagaard, B. T., Knepley, M. G., \& Williams, C. A., 2017. Pylith user manual: Version 2.2.1, Davis, CA: Computational Infrastructure of Geodynamics.

Adams, B. M., Bohnhoff, W. J., Dalbey, K. R., Eddy, J. P., Eldred, M. S., Gay, D. M., Haskell, K., Hough, P. D., \& Swiler, L. P., 2009. DAKOTA, a multilevel parallel object-oriented framework for design optimization, parameter estimation, uncertainty quantification, and sensitivity analysis: version 5.0 user's manual, Sandia National Laboratories, Tech. Rep. SAND2010-2183.

Alexanderian, A., Gloor, P. J., Ghattas, O., et al., 2016. On Bayesian A-and D-optimal experimental designs in infinite dimensions, Bayesian Analysis, 11(3), 671-695.

Alghamdi, A., Hesse, M. A., Chen, J., \& Ghattas, O., 2020. Bayesian poroelastic aquifer characterization from InSAR surface deformation data. part I: Maximum a posteriori estimate, Water Resour. Res., 56(10), e2020WR027391.

Alnæs, M. S., Logg, A., Ølgaard, K. B., Rognes, M. E., \& Wells, G. N., 2014. Unified form language: A domain-specific language for weak formulations of partial differential equations, ACM Trans. Math. Software (TOMS), 40(2), 1-37. 
Ambartsumyan, I., Khattatov, E., Nordbotten, J. M., \& Yotov, I., 2020. A multipoint stress mixed finite element method for elasticity on simplicial grids, SIAM J. Num. Analys., 58(1), 630-656.

Amestoy, P. R., Duff, I. S., L'Excellent, J.-Y., \& Koster, J., 2001. A fully asynchronous multifrontal solver using distributed dynamic scheduling, SIAM J. Matrix Analys. Applic., 23(1), 15-41.

Amestoy, P. R., Buttari, A., L'excellent, J.-Y., \& Mary, T., 2019. Performance and scalability of the block low-rank multifrontal factorization on multicore architectures, ACM Trans. Math. Software (TOMS), 45(1), $1-26$.

Arnold, D., Falk, R., \& Winther, R., 2007. Mixed finite element methods for linear elasticity with weakly imposed symmetry, Math. Comput., 76(260), 1699-1723.

Arnold, D. N., 1990. Mixed finite element methods for elliptic problems, Comp. Meth. Appl. Mech. Eng., 82(1-3), 281-300.

Arnold, D. N. \& Brezzi, F., 1985. Mixed and nonconforming finite element methods: implementation, postprocessing and error estimates, ESAIM: Mathematical Modelling and Numerical Analysis-Modélisation Mathématique et Analyse Numérique, 19(1), 7-32.

Arnold, D. N., Brezzi, F., \& Douglas, J., 1984a. PEERS: a new mixed finite element for plane elasticity, Japan J. Appl. Math., 1(2), 347-367.

Arnold, D. N., Douglas, J., \& Gupta, C. P., 1984b. A family of higher order mixed finite element methods for plane elasticity, Numerische Mathematik, 45(1), 1-22.

Babaniyi, O., Nicholson, R., Villa, U., \& Petra, N., 2021. Inferring the basal sliding coefficient field for the stokes ice sheet model under rheological uncertainty, The Cryosphere, 15(4), 1731-1750.

Bærland, T., Lee, J. J., Mardal, K.-A., \& Winther, R., 2017. Weakly imposed symmetry and robust preconditioners for Biot's consolidation model, Comp. Methods Appl. Math., 17(3), 377-396.

Balay, S., Gropp, W. D., McInnes, L. C., \& Smith, B. F., 1997. Efficient management of parallelism in object-oriented numerical software libraries, in Modern software tools for scientific computing, pp. 163-202, Springer.

Balay, S., Abhyankar, S., Adams, M. F., Brown, J., Brune, P., Buschelman, K., Dalcin, L., Dener, A., Eijkhout, V., Gropp, W., et al., 2020. PETSc users manual revision 3.13, Tech. rep., Argonne National Lab.(ANL), Argonne, IL (United States).

Bangerth, W., Hartmann, R., \& Kanschat, G., 2007. deal. II—a general-purpose object-oriented finite element library, ACM Trans. Math. Software (TOMS), 33(4), 24-es.

Barbot, S., 2014. RELAX v1.0.7, Computational Infrastructure for Geodynamics, geodynamics.org.

Bartlow, N. M., Wallace, L. M., Beavan, R. J., Bannister, S., \& Segall, P., 2014. Time-dependent modeling of slow slip events and associated seismicity and tremor at the Hikurangi subduction zone, New Zealand, $J$. geophys. Res., 119(1), 734-753.

Bashir, O., Willcox, K., Ghattas, O., van Bloemen Waanders, B., \& Hill, J., 2008. Hessian-based model reduction for large-scale systems with initial-condition inputs, Int. J. Numer. Meth. Eng., 73(6), 844-868.

Baumann, T. S. \& Kaus, B. J. P., 2015. Geodynamic inversion to constrain the non-linear rheology of the 
lithosphere, Geophys. J Int., 202(2), 1289-1316.

Baumann, T. S., Kaus, B. J. P., \& Popov, A. A., 2014. Constraining effective rheology through parallel joint geodynamic inversion, Tectonophysics, 631, 197-211.

Becker, T. W., Hashima, A., Freed, A. M., \& Sato, H., 2018. Stress change before and after the 2011 M9 Tohoku-oki earthquake, Earth Planet. Sci. Lett., 504, 174-184.

Beskos, A., Girolami, M., Lan, S., Farrell, P. E., \& Stuart, A. M., 2017. Geometric MCMC for infinitedimensional inverse problems, J. Comput. Physics, 335, 327-351.

Boffi, D., Brezzi, F., \& Fortin, M., 2009. Reduced symmetry elements in linear elasticity, Comm. Pure Appl. Analys., 8(1), 95.

Brezzi, F., 1974. On the existence, uniqueness and approximation of saddle-point problems arising from Lagrangian multipliers, Publications mathématiques et informatique de Rennes, (S4), 1-26.

Brezzi, F., Douglas, J., \& Marini, L. D., 1985. Two families of mixed finite elements for second order elliptic problems, Numerische Mathematik, 47(2), 217-235.

Bui-Thanh, T. \& Ghattas, O., 2013. Analysis of the hessian for inverse scattering problems: Part III: Inverse medium scattering of electromagnetic waves in three dimensions, Inverse Probl. Imaging, 7(4).

Bui-Thanh, T. \& Ghattas, O., 2015. A scalable algorithm for MAP estimators in Bayesian inverse problems with Besov priors, Inverse Probl. Imaging, 9(1), 27.

Bui-Thanh, T., Burstedde, C., Ghattas, O., Martin, J., Stadler, G., \& Wilcox, L. C., 2012. Extreme-scale UQ for Bayesian inverse problems governed by PDEs, in SC'12: Proceedings of the international conference on high performance computing, networking, storage and analysis, pp. 1-11, IEEE.

Bui-Thanh, T., Ghattas, O., Martin, J., \& Stadler, G., 2013. A computational framework for infinitedimensional bayesian inverse problems part I: The linearized case, with application to global seismic inversion, SIAM J. Scient. Comp., 35(6), A2494-A2523.

Chen, P., Wu, K., Chen, J., O’Leary-Roseberry, T., \& Ghattas, O., 2019. Projected Stein variational Newton: A fast and scalable Bayesian inference method in high dimensions, arXiv preprint arXiv:1901.08659.

Ciarlet, P. G., 2002. The finite element method for elliptic problems, SIAM.

Cockburn, B., Gopalakrishnan, J., \& Guzmán, J., 2010. A new elasticity element made for enforcing weak stress symmetry, Math. Comput., 79(271), 1331-1349.

Dagum, L. \& Menon, R., 1998. OpenMP: an industry standard API for shared-memory programming, IEEE Comput. Sci. Eng., 5(1), 46-55.

Damiani, L. H., Kosakowski, G., Glaus, M. A., \& Churakov, S. V., 2020. A framework for reactive transport modeling using FEniCS-Reaktoro: governing equations and benchmarking results, Comput. Geosci., pp. 115.

Dragert, H., Wang, K., \& James, T. S., 2001. A silent slip event on the deeper Cascadia subduction interface, Science, 292(5521), 1525-1528.

Ellis, S., Fagereng, Å., Barker, D., Henrys, S., Saffer, D., Wallace, L., Williams, C., \& Harris, R., 2015. Fluid budgets along the northern Hikurangi subduction margin, New Zealand: The effect of a subducting seamount 
on fluid pressure, Geophys. J Int., 202(1), 277-297.

Engl, H. W., Hanke, M., \& Neubauer, A., 1996. Regularization of inverse problems, vol. 375, Springer Science \& Business Media.

Erickson, B. A., Jiang, J., Barall, M., Lapusta, N., Dunham, E. M., Harris, R., Abrahams, L. S., Allison, K. L., Ampuero, J.-P., Barbot, S., et al., 2020. The community code verification exercise for simulating sequences of earthquakes and aseismic slip (SEAS), Seism. Res. Lett., 91(2A), 874-890.

Falk, R. S., 2008. Finite element methods for linear elasticity, in Mixed finite elements, compatibility conditions, and applications, pp. 159-194, Springer.

Farhloul, M. \& Fortin, M., 1997. Dual hybrid methods for the elasticity and the Stokes problems: a unified approach, Numerische Mathematik, 76(4), 419-440.

Farrell, P. E., Ham, D. A., Funke, S. W., \& Rognes, M. E., 2013. Automated derivation of the adjoint of high-level transient finite element programs, SIAM J. Scient. Comp., 35(4), C369-C393.

Flath, H. P., Wilcox, L. C., Akçelik, V., Hill, J., van Bloemen Waanders, B., \& Ghattas, O., 2011. Fast algorithms for Bayesian uncertainty quantification in large-scale linear inverse problems based on low-rank partial Hessian approximations, SIAM J. Scient. Comp., 33(1), 407-432.

Fraeijs de Veubeke, B., 1965. Displacement and equilibrium models in the finite element method, Stress analysis, pp. chapter-9.

Fraeijs de Veubeke, B., 1975. Stress function approach, Tech. rep., LTAS.

Gallovič, F., Valentová, L., Ampuero, J. P., \& Gabriel, A. A., 2019. Bayesian dynamic finite-fault inversion: 2. Application to the $2016 \mathrm{Mw} 6.2$ Amatrice, Italy, earthquake, J. geophys. Res., 124(7), 6970-6988.

Geuzaine, C. \& Remacle, J.-F., 2009. Gmsh: A 3-D finite element mesh generator with built-in pre-and postprocessing facilities, Int. J. Num. Meth. Eng., 79(11), 1309-1331.

Ghattas, O. \& Willcox, K., 2021. Learning physics-based models from data: perspectives from inverse problems and model reduction, Acta Numerica, 30, 445-554.

Gropp, W., Gropp, W. D., Lusk, E., Skjellum, A., \& Lusk, A., 1999. Using MPI: portable parallel programming with the message-passing interface, vol. 1, MIT press.

Haagenson, R., Rajaram, H., \& Allen, J., 2020. A generalized poroelastic model using FEniCS with insights into the Noordbergum effect, Computers \& Geosciences, 135, 104399.

Hadamard, J., 1923. Lectures on Cauchy's problem in linear partial differential equations, Press. New Haven. Halko, N., Martinsson, P., \& Tropp, J. A., 2011. Finding structure with randomness: Probabilistic algorithms for constructing approximate matrix decompositions, SIAM review, 53(2), 217-288.

Ham, D. A., Farrell, P. E., Gorman, G. J., Maddison, J. R., Wilson, C. R., Kramer, S. C., Shipton, J., Collins, G. S., Cotter, C. J., \& Piggott, M. D., 2009. Spud 1.0: generalising and automating the user interfaces of scientific computer models, Geoscientific Model Development, 2(1), 33-42.

Heroux, M. A., Bartlett, R. A., Howle, V. E., Hoekstra, R. J., Hu, J. J., Kolda, T. G., Lehoucq, R. B., Long, K. R., Pawlowski, R. P., Phipps, E. T., et al., 2005. An overview of the Trilinos project, ACM Trans. Math. Software (TOMS), 31(3), 397-423. 
Hesse, M. A. \& Stadler, G., 2014. Joint inversion in coupled quasi-static poroelasticity, J. geophys. Res., 119(2), 1425-1445.

Hsu, Y.-J., Simons, M., Avouac, J.-P., Galetzka, J., Sieh, K., Chlieh, M., Natawidjaja, D., Prawirodirdjo, L., \& Bock, Y., 2006. Frictional afterslip following the 2005 nias-simeulue earthquake, sumatra, Science, 312(5782), 1921-1926.

Hsu, Y.-J., Simons, M., Williams, C., \& Casarotti, E., 2011. Three-dimensional FEM derived elastic Green's functions for the coseismic deformation of the $2005 \mathrm{Mw} 8.7$ Nias-Simeulue, Sumatra earthquake, Geochem. Geophys. Geosys., 12(7).

Im, K., Saffer, D., Marone, C., \& Avouac, J.-P., 2020. Slip-rate-dependent friction as a universal mechanism for slow slip events, Nat. Geosci., 13(10), 705-710.

Isaac, T., Petra, N., Stadler, G., \& Ghattas, O., 2015. Scalable and efficient algorithms for the propagation of uncertainty from data through inference to prediction for large-scale problems, with application to flow of the Antarctic ice sheet, J. Comput. Phys., 296, 348-368.

Ito, Y., Hino, R., Kido, M., Fujimoto, H., Osada, Y., Inazu, D., Ohta, Y., Iinuma, T., Ohzono, M., \& Miura, S., 2013. Episodic slow slip events in the Japan subduction zone before the 2011 Tohoku-oki earthquake, Tectonophysics, 600, 14-26.

Jackson, D. D., 1979. The use of a priori data to resolve non-uniqueness in linear inversion, Geophys. J Int., 57(1), 137-157.

Johnson, C., 1977. A mixed finite element method for plasticity problems with hardening, SIAM J. Num. Analys., 14(4), 575-583.

Khattatov, E. \& Yotov, I., 2019. Domain decomposition and multiscale mortar mixed finite element methods for linear elasticity with weak stress symmetry, ESAIM: Math. Model. Num. Analys., 53(6), 2081-2108.

Kirby, R. C. \& Logg, A., 2006. A compiler for variational forms, ACM Trans. Math. Software (TOMS), 32(3), 417-444.

Klawonn, A. \& Starke, G., 2004. A preconditioner for the equations of linear elasticity discretized by the PEERS element, Numerical linear algebra with applications, 11(5-6), 493-510.

Lawn, B. R. \& Wilshaw, T. R., 1975. Fracture of brittle solids, Cambridge University Press.

Lawson, C. L. \& Hanson, R. J., 1995. Solving least squares problems, SIAM.

Liu, C., Lay, T., Brodsky, E. E., Dascher-Cousineau, K., \& Xiong, X., 2019. Coseismic rupture process of the large 2019 Ridgecrest earthquakes from joint inversion of geodetic and seismological observations, Geophys. Res. Lett., 46(21), 11820-11829.

Liu, P. \& Archuleta, R. J., 2004. A new nonlinear finite fault inversion with three-dimensional Green's functions: Application to the 1989 Loma Prieta, California, earthquake, J. geophys. Res., 109(B2).

Logg, A. \& Wells, G. N., 2010. DOLFIN: Automated finite element computing, ACM Trans. Math. Software (TOMS), 37(2), 20.

Logg, A., Wells, G. N., \& Hake, J., 2012. DOLFIN: A C++/Python finite element library, in Automated solution of differential equations by the finite element method, pp. 173-225, Springer. 
Martin, J., Wilcox, L. C., Burstedde, C., \& Ghattas, O., 2012. A stochastic Newton MCMC method for large-scale statistical inverse problems with application to seismic inversion, SIAM J. Scient. Comp., 34(3), A1460-A1487.

Mavrommatis, A. P., Segall, P., \& Johnson, K. M., 2014. A decadal-scale deformation transient prior to the 2011 Mw 9.0 Tohoku-oki earthquake, Geophys. Res. Lett., 41(13), 4486-4494.

McCormack, K. A. et al., 2018. Earthquakes, groundwater and surface deformation: exploring the poroelastic response to megathrust earthquakes, Ph.D. thesis.

McCormack, K. A. \& Hesse, M. A., 2018. Modeling the poroelastic response to megathrust earthquakes: A look at the 2012 Mw 7.6 Costa Rican event, Adv. Water Resources, 114, 236-248.

McDougall, D., Malaya, N., \& Moser, R. D., 2017. The parallel C++ statistical library for bayesian inference: Queso, Springer International Publishing, pp. 1829-1865.

Melosh, H. J. \& Raefsky, A., 1981. A simple and efficient method for introducing faults into finite element computations, Bull. seism. Soc. Am., 71(5), 1391-1400.

Menke, W., 2018. Geophysical data analysis: Discrete inverse theory, Academic press.

Miller, K., 1970. Least squares methods for ill-posed problems with a prescribed bound, SIAM J. Math. Analys., 1(1), 52-74.

Multiphysics, C., 1998. Introduction to comsol multiphysics@, COMSOL Multiphysics, Burlington, MA, accessed Feb, 9, 2018.

Nanjo, K. Z., Hirata, N., Obara, K., \& Kasahara, K., 2012. Decade-scale decrease in b value prior to the M9-class 2011 Tohoku and 2004 Sumatra quakes, Geophys. Res. Lett., 39(20).

Nédélec, J.-C., 1986. A new family of mixed finite elements in $\mathbb{R}^{3}$, Numerische Mathematik, 50(1), 57-81.

Oberkampf, W. L. \& Roy, C. J., 2010. Verification and validation in scientific computing, Cambridge University Press.

Okada, Y., 1985. Surface deformation due to shear and tensile faults in a half-space, Bull. seism. Soc. Am., 75(4), 1135-1154.

Okada, Y., 1992. Internal deformation due to shear and tensile faults in a half-space, Bull. seism. Soc. Am., 82(2), 1018-1040.

Ozawa, S., Nishimura, T., Munekane, H., Suito, H., Kobayashi, T., Tobita, M., \& Imakiire, T., 2012. Preceding, coseismic, and postseismic slips of the 2011 Tohoku earthquake, Japan, J. geophys. Res., 117(B7).

Parno, M., Davis, A., Seelinger, L., \& Marzouk, Y., 2014. MIT uncertainty quantification (MUQ) library.

Pellegrini, F., 2008. Scotch and libScotch 5.1 user's guide.

Petra, N., Martin, J., Stadler, G., \& Ghattas, O., 2014. A computational framework for infinite-dimensional Bayesian inverse problems, Part II: Stochastic Newton MCMC with application to ice sheet flow inverse problems, SIAM J. Scient. Comp., 36(4), A1525-A1555.

Phillips, D. L., 1962. A technique for the numerical solution of certain integral equations of the first kind, Journal of the ACM (JACM), 9(1), 84-97.

Pollard, D. D. \& Segall, P., 1987. Theoretical displacements and stresses near fractures in rock: with applica- 
tions to faults, joints, veins, dikes, and solution surfaces, in Fracture mechanics of rock, pp. 277-347.

Pritchard, M. E., Allen, R. M., Becker, T. W., Behn, M. D., Brodsky, E. E., Bürgmann, R., Ebinger, C., Freymueller, J. T., Gerstenberger, M., Haines, B., et al., 2020. New opportunities to study earthquake precursors. Project Jupyter, 2021. Jupyter website, online at jupyter. org/index. html, accessed 01/2021.

Rathgeber, F., Ham, D. A., Mitchell, L., Lange, M., Luporini, F., McRae, A. T. T., Bercea, G.-T., Markall, G. R., \& Kelly, P. H. J., 2016. Firedrake: automating the finite element method by composing abstractions, ACM Trans. Math. Software (TOMS), 43(3), 1-27.

Rees, T. \& Wathen, M., 2020. An element-based preconditioner for mixed finite element problems, SIAM J. Scient. Comp..

Rhebergen, S., Wells, G. N., Wathen, A. J., \& Katz, R. F., 2015. Three-field block preconditioners for models of coupled magma/mantle dynamics, SIAM J. Scient. Comp., 37(5), A2270-A2294.

Roache, P. J., 2002. Code verification by the method of manufactured solutions, J. Fluids Eng., 124(1), 4-10.

Rognes, M. E. \& Winther, R., 2010. Mixed finite element methods for linear viscoelasticity using weak symmetry, Math. Models Meth. Appl. Sci, 20(06), 955-985.

Ruiz, S., Metois, M., Fuenzalida, A., Ruiz, J., Leyton, F., Grandin, R., Vigny, C., Madariaga, R., \& Campos, J., 2014. Intense foreshocks and a slow slip event preceded the 2014 Iquique Mw 8.1 earthquake, Science, 345(6201), 1165-1169.

Ruthotto, L., Treister, E., \& Haber, E., 2017. jInv-a flexible Julia package for PDE parameter estimation, SIAM J. Scient. Comp., 39(5), S702-S722.

Scholz, C. H., 2019. The mechanics of earthquakes and faulting, Cambridge university press.

Segall, P., 2010. Earthquake and volcano deformation, Princeton University Press.

Simpson, G., Spiegelman, M., \& Weinstein, M. I., 2010. A multiscale model of partial melts: 2. Numerical results, J. geophys. Res., 115(B4).

Smith, M., 2009. ABAQUS/Standard User's Manual, Version 6.9, Dassault Systèmes Simulia Corp, United States.

Spiegelman, M., May, D. A., \& Wilson, C. R., 2016. On the solvability of incompressible Stokes with viscoplastic rheologies in geodynamics, Geochem. Geophys. Geosys., 17(6), 2213-2238.

Stenberg, R., 1984. Analysis of mixed finite elements methods for the Stokes problem: a unified approach, Math. Comp., 42(165), 9-23.

Stenberg, R., 1988. A family of mixed finite elements for the elasticity problem, Numerische Mathematik, 53(5), 513-538.

Sun, T., Saffer, D., \& Ellis, S., 2020. Mechanical and hydrological effects of seamount subduction on megathrust stress and slip, Nat. Geosci., 13(3), 249-255.

Tada, H., Paris, P. C., \& Irwin, G. R., 1973. The stress analysis of cracks handbook, del res, Corp., Hellertown, $P a$.

Tarantola, A., 2005. Inverse problem theory and methods for model parameter estimation, SIAM.

Tikhonov, A. N., 1963. Solution of incorrectly formulated problems and the regularization method, Soviet 
Math., 4, 1035-1038.

Tikhonov, A. N. \& Arsenin, V. Y., 1977. Solutions of ill-posed problems.

Tong, C., 2017. Problem solving environment for uncertainty analysis and design exploration, Tech. rep., Lawrence Livermore National Lab.(LLNL), Livermore, CA (United States).

Tosi, N., Stein, C., Noack, L., Hüttig, C., Maierova, P., Samuel, H., Davies, D. R., Wilson, C. R., Kramer, S. C., Thieulot, C., et al., 2015. A community benchmark for viscoplastic thermal convection in a 2-D square box, Geochem. Geophys. Geosys., 16(7), 2175-2196.

Tröltzsch, F., 2010. Optimal control of partial differential equations: theory, methods, and applications, vol. 112, American Mathematical Soc.

Uchida, N. \& Bürgmann, R., 2021. A decade of lessons learned from the 2011 Tohoku-oki earthquake, Rev. Geophys., 59(2), e2020RG000713.

Uchida, N. \& Matsuzawa, T., 2013. Pre-and postseismic slow slip surrounding the 2011 Tohoku-oki earthquake rupture, Earth Planet. Sci. Lett., 374, 81-91.

van Keken, P. E., Currie, C., King, S. D., Behn, M. D., Cagnioncle, A., He, J., Katz, R. F., Lin, S., Parmentier, E. M., Spiegelman, M., et al., 2008. A community benchmark for subduction zone modeling, Phys. Earth Planet. Inter., 171(1-4), 187-197.

Villa, U., Petra, N., \& Ghattas, O., 2016. hIPPYlib: an Extensible Software Framework for Large-scale Deterministic and Bayesian Inverse Problems.

Villa, U., Petra, N., \& Ghattas, O., 2018. hIPPYlib: an Extensible Software Framework for Large-scale Deterministic and Bayesian Inverse Problems, J. Open Source Software, 3(30).

Villa, U., Petra, N., \& Ghattas, O., 2021. hIPPYlib: An Extensible Software Framework for Large-Scale Inverse Problems Governed by PDEs; Part I: Deterministic Inversion and Linearized Bayesian Inference, ACM Trans. Math. Software (TOMS), 47(2), 1-34.

Vogel, C. R., 2002. Computational methods for inverse problems, SIAM.

Vynnytska, L., Rognes, M. E., \& Clark, S. R., 2013. Benchmarking FEniCS for mantle convection simulations, Computers \& Geosciences, 50, 95-105.

Wallace, L. M., 2020. Slow slip events in New Zealand, Ann. Rev. Earth Planet Sci., 48, 175-203.

Wallace, L. M., Webb, S. C., Ito, Y., Mochizuki, K., Hino, R., Henrys, S., Schwartz, S. Y., \& Sheehan, A. F., 2016. Slow slip near the trench at the Hikurangi subduction zone, New Zealand, Science, 352(6286), 701704.

Wang, K., Bui-Thanh, T., \& Ghattas, O., 2018. A randomized maximum a posteriori method for posterior sampling of high dimensional nonlinear Bayesian inverse problems, SIAM J. Scient. Comp., 40(1), A142A171.

Wang, K., Dreger, D. S., Tinti, E., Bürgmann, R., \& Taira, T., 2020. Rupture process of the 2019 Ridgecrest, California Mw 6.4 foreshock and Mw 7.1 earthquake constrained by seismic and geodetic data, Bull. seism. Soc. Am., 110(4), 1603-1626.

Wang, Q., Campillo, M., Brenguier, F., Lecointre, A., Takeda, T., \& Hashima, A., 2019. Evidence of changes 
of seismic properties in the entire crust beneath Japan after the Mw 9.0, 2011 Tohoku-oki earthquake, $J$. geophys. Res..

Wang, Q., Campillo, M., Brenguier, F., Lecointre, A., Takeda, T., \& Yoshida, K., 2021. Seismic evidence of fluid migration in northeastern Japan after the 2011 Tohoku-oki earthquake, Earth Planet. Sci. Lett., 563, 116894.

Wildey, T. \& Xue, G., 2013. Preconditioning for mixed finite element formulations of elliptic problems, in Domain Decomposition Methods in Science and Engineering XX, pp. 175-182, Springer.

Williams, C. A. \& Wallace, L. M., 2015. Effects of material property variations on slip estimates for subduction interface slow-slip events, Geophys. Res. Lett., 42(4), 1113-1121.

Wilson, C. \& Spiegelman, M., 2016. TerraFERMA benchmarks, Tech. rep., technical report, Columbia Univ., New York, NY, doi: 10.6084/m9.

Wilson, C. R., Spiegelman, M., van Keken, P. E., \& Hacker, B. R., 2014. Fluid flow in subduction zones: The role of solid rheology and compaction pressure, Earth Planet. Sci. Lett., 401, 261-274.

Wilson, C. R., Spiegelman, M., \& van Keken, P. E., 2017. TerraFERMA: The Transparent Finite Element Rapid Model Assembler for multiphysics problems in Earth sciences, Geochem. Geophys.,Geosys., 18(2), 769-810.

Worthen, J., Stadler, G., Petra, N., Gurnis, M., \& Ghattas, O., 2014. Towards adjoint-based inversion for rheological parameters in nonlinear viscous mantle flow, Phys. Earth Planet. Inter., 234, 23-34.

Yokota, Y. \& Koketsu, K., 2015. A very long-term transient event preceding the 2011 Tohoku earthquake, Nat. Commun., 6, 5934.

Yoshida, K., Hasegawa, A., Okada, T., Iinuma, T., Ito, Y., \& Asano, Y., 2012. Stress before and after the 2011 great Tohoku-oki earthquake and induced earthquakes in inland areas of eastern Japan, Geophys. Res. Lett., 39(3).

\section{APPENDIX A: DERIVATION OF MANUFACTURED SOLUTION}

We derive the expression of the source term $\boldsymbol{f}(x, y)$ and exact stress given an exact solution, using the method of manufactured solution (Roache 2002; Oberkampf \& Roy 2010). Considering a 2D connected domain $\Omega \subset \mathbb{R}^{2}$ with boundaries $\partial \Omega=\Gamma_{D}$, the boundary value problem of the linear elasticity in the pure displacement formulation, with vanishing Dirichlet boundaries, reads

$$
\begin{cases}-\nabla \cdot \boldsymbol{\sigma}=\boldsymbol{f} & \text { in } \Omega, \\ \boldsymbol{\sigma}=2 \mu \boldsymbol{\varepsilon}+\lambda \operatorname{tr}(\boldsymbol{\varepsilon}) \boldsymbol{I} & \text { in } \Omega, \\ \boldsymbol{\varepsilon}=\frac{1}{2}\left(\nabla \boldsymbol{u}+(\nabla \boldsymbol{u})^{T}\right) & \text { in } \Omega, \\ \boldsymbol{u}=0 & \text { on } \Gamma_{D} .\end{cases}
$$


Let the exact solution be given by

$\boldsymbol{u}_{\mathrm{ex}}=\left(\begin{array}{c}0 \\ \sin (2 \pi x) \sin (2 \pi y)\end{array}\right)$

We want to determine the source term $\boldsymbol{f}(x, y)$ and the boundary conditions, such that they give the exact solution. In order to find the source term $\boldsymbol{f}(x, y)$, we substitute the expression of the strain tensor $\varepsilon$ into the conservation of linear momentum equation to get

$\nabla \cdot\left(\mu\left(\nabla \boldsymbol{u}_{\mathrm{ex}}+\nabla \boldsymbol{u}_{\mathrm{ex}}^{T}\right)+\lambda \nabla \cdot \boldsymbol{u}_{\mathrm{ex}} \boldsymbol{I}\right)=\boldsymbol{f}$.

Remember that for a vector-value function $\boldsymbol{f}(x, y)$ the gradient can be written as

$\nabla \boldsymbol{f}=\left(\begin{array}{cc}\frac{\partial}{\partial x} f_{x} & \frac{\partial}{\partial x} f_{y} \\ \frac{\partial}{\partial y} f_{x} & \frac{\partial}{\partial y} f_{y}\end{array}\right)$

Therefore, substituting eq. (A.2) into eq. (A.3), we can write

$$
\begin{aligned}
& \nabla \cdot\left[\mu\left(\begin{array}{ll}
0 & 2 \pi \cos (2 \pi x) \sin (2 \pi y) \\
0 & 2 \pi \sin (2 \pi x) \cos (2 \pi y)
\end{array}\right)+\left(\begin{array}{cc}
0 & 0 \\
2 \pi \cos (2 \pi x) \sin (2 \pi y) & 2 \pi \sin (2 \pi x) \cos (2 \pi y)
\end{array}\right)\right. \\
& \left.+\lambda\left(\begin{array}{cc}
2 \pi \sin (2 \pi x) \cos (2 \pi y) & 0 \\
0 & 2 \pi \sin (2 \pi x) \cos (2 \pi y)
\end{array}\right)\right]=\boldsymbol{f}, \\
& \nabla \cdot\left[\mu\left(\begin{array}{cc}
0 & 2 \pi \cos (2 \pi x) \sin (2 \pi y) \\
2 \pi \cos (2 \pi x) \sin (2 \pi y) & 4 \pi \sin (2 \pi x) \cos (2 \pi y)
\end{array}\right)\right. \\
& \left.+\lambda\left(\begin{array}{cc}
2 \pi \sin (2 \pi x) \cos (2 \pi y) & 0 \\
0 & 2 \pi \sin (2 \pi x) \cos (2 \pi y)
\end{array}\right)\right]=\boldsymbol{f}, \\
& \nabla \cdot\left(\begin{array}{cc}
\lambda 2 \pi \sin (2 \pi x) \cos (2 \pi y) & \mu 2 \pi \cos (2 \pi x) \sin (2 \pi y) \\
\mu 2 \pi \cos (2 \pi x) \sin (2 \pi y) & (2 \mu+\lambda) 2 \pi \sin (2 \pi x) \cos (2 \pi y)
\end{array}\right)=\boldsymbol{f}
\end{aligned}
$$

Now, we know that the divergence of a tensor-valued function $\boldsymbol{F}$ can be written as

$\nabla \cdot \boldsymbol{F}=\left(\begin{array}{ll}\frac{\partial}{\partial x} F_{x x} & \frac{\partial}{\partial y} F_{x y} \\ \frac{\partial}{\partial x} F_{y x} & \frac{\partial}{\partial y} F_{y y}\end{array}\right)$

Therefore, we can write the expression as

$$
\left(\begin{array}{c}
\lambda 4 \pi^{2} \cos (2 \pi x) \cos (2 \pi y)+\mu 4 \pi^{2} \cos (2 \pi x) \cos (2 \pi y) \\
-\mu 4 \pi^{2} \sin (2 \pi x) \sin (2 \pi y)-(2 \mu+\lambda) 4 \pi^{2} \sin (2 \pi x) \sin (2 \pi y)
\end{array}\right)=\boldsymbol{f} .
$$


The source term $\boldsymbol{f}(x, y)$ can be finally written as

$\boldsymbol{f}(x, y)=\left(\begin{array}{c}4 \pi^{2}(\mu+\lambda) \cos (2 \pi x) \cos (2 \pi y) \\ -4 \pi^{2}(3 \mu+\lambda) \sin (2 \pi x) \sin (2 \pi y)\end{array}\right)$.

This is the exact source term which produces the exact solution (eq. A.2). The vanishing Dirichlet boundary conditions $\boldsymbol{u}=0$ on $\Gamma_{D}$ remain the same. Finally, the exact stress can be written as

$\boldsymbol{\sigma}_{\mathrm{ex}}=\left(\begin{array}{cc}\lambda 2 \pi \sin (2 \pi x) \cos (2 \pi y) & \mu 2 \pi \cos (2 \pi x) \sin (2 \pi y) \\ \mu 2 \pi \cos (2 \pi x) \sin (2 \pi y) & (2 \mu+\lambda) 2 \pi \sin (2 \pi x) \cos (2 \pi y)\end{array}\right)$.

\section{APPENDIX B: ANALYTIC EXPRESSIONS FOR MODE II CRACK}

In this appendix we provide the analytic expressions of the displacement and stress fields for a crack mode II following Pollard \& Segall (1987) and used in this study. We consider a 2D domain and a shear crack of unity width, $2 a=1$, where $a$ is the half-width of the crack. The formulas below are expressed in polar coordinates centred at the crack middle $\left(x_{0}, y_{0}\right)$ and tips $a$. Following Pollard \& Segall (1987), we can define the polar coordinates as (Pollard \& Segall 1987, eqs 8.29 and 8.31)

$$
\begin{aligned}
\boldsymbol{r}_{1} & =\sqrt{\left[x-\left(x_{0}-a\right)\right]^{2}+\left(y-y_{0}\right)^{2}}, & \boldsymbol{\theta}_{1} & =\arctan \left[\frac{y-y_{0}}{x-\left(x_{0}-a\right)}\right], \\
\boldsymbol{r}_{2} & =\sqrt{\left[x-\left(x_{0}+a\right)\right]^{2}+\left(y-y_{0}\right)^{2}}, & \boldsymbol{\theta}_{2} & =\arctan \left[\frac{y-y_{0}}{x-\left(x_{0}+a\right)}\right], \\
\boldsymbol{r} & =\sqrt{\left(x-x_{0}\right)^{2}+\left(y-y_{0}\right)^{2}}, & \boldsymbol{\theta} & =\arctan \left[\frac{y-y_{0}}{x-x_{0}}\right], \\
\boldsymbol{R} & =\sqrt{\boldsymbol{r}_{1} \boldsymbol{r}_{2}}, & \boldsymbol{\Theta} & =\frac{\boldsymbol{\theta}_{1}+\boldsymbol{\theta}_{2}}{2} .
\end{aligned}
$$

Given a shear driving stress $\Delta \sigma_{I I}$ and an elastic material with shear modulus $\mu$, the two components of the displacement field caused by relative motion of the crack walls are (Pollard \& Segall 1987, eq. 8.33)

$$
\begin{aligned}
& u_{x}=\frac{\Delta \sigma_{I I}}{2 \mu}\left\{2(1-\nu)[\boldsymbol{R} \sin \boldsymbol{\Theta}-\boldsymbol{r} \sin \boldsymbol{\theta}]+\boldsymbol{r} \sin \boldsymbol{\theta}\left[\frac{\boldsymbol{r}}{\boldsymbol{R}} \cos (\boldsymbol{\theta}-\boldsymbol{\Theta})-1\right]\right\}, \\
& u_{y}=-\frac{\Delta \sigma_{I I}}{2 \mu}\left\{(1-2 \nu)[\boldsymbol{R} \cos \boldsymbol{\Theta}-\boldsymbol{r} \cos \boldsymbol{\theta}]+\boldsymbol{r} \sin \boldsymbol{\theta}\left[\frac{\boldsymbol{r}}{\boldsymbol{R}} \sin (\boldsymbol{\theta}-\boldsymbol{\Theta})\right]\right\} .
\end{aligned}
$$

We apply a unity driving stress, which leads to an elliptical solution for slip in the form of (Pollard \& Segall 1987, eq. 8.34)

$\Delta u=\Delta \sigma_{I I} \frac{1-\nu}{\mu} \sqrt{a^{2}-\left(\boldsymbol{x}_{\mathrm{crack}}-x_{0}\right)^{2}}$,

where $\nu$ is the Poisson's ratio of the elastic material, and $\boldsymbol{x}_{\text {crack }}$ is the x-coordinate along the crack such that $\left|\boldsymbol{x}_{\text {crack }}\right| \leq a$. Lastly, the three independent components of the stress field can be written as 
(Pollard \& Segall 1987, eq. 8.44)

$$
\begin{aligned}
\sigma_{x x} & =\sigma_{x x}^{r}+\Delta \sigma_{I I}\left[\frac{2 \boldsymbol{r}}{\boldsymbol{R}} \sin (\boldsymbol{\theta}-\boldsymbol{\Theta})-a^{2} \frac{\boldsymbol{r}}{\boldsymbol{R}^{3}} \sin \boldsymbol{\theta} \cos (3 \boldsymbol{\Theta})\right], \\
\sigma_{x y} & =\sigma_{x y}^{r}+\Delta \sigma_{I I}\left[\frac{\boldsymbol{r}}{\boldsymbol{R}} \cos (\boldsymbol{\theta}-\boldsymbol{\Theta})-1-a^{2} \frac{\boldsymbol{r}}{\boldsymbol{R}^{3}} \sin \boldsymbol{\theta} \sin (3 \boldsymbol{\Theta})\right], \\
\sigma_{y y} & =\sigma_{y y}^{r}+\Delta \sigma_{I I}\left[a^{2} \frac{\boldsymbol{r}}{\boldsymbol{R}^{3}} \sin \boldsymbol{\theta} \cos (3 \boldsymbol{\Theta})\right],
\end{aligned}
$$

where $\sigma_{x x}^{r}, \sigma_{x y}^{r}$ and $\sigma_{y y}^{r}$ are the corresponding remote/background stresses that act even in absence of the crack. 


\section{SUPPORTING INFORMATION}

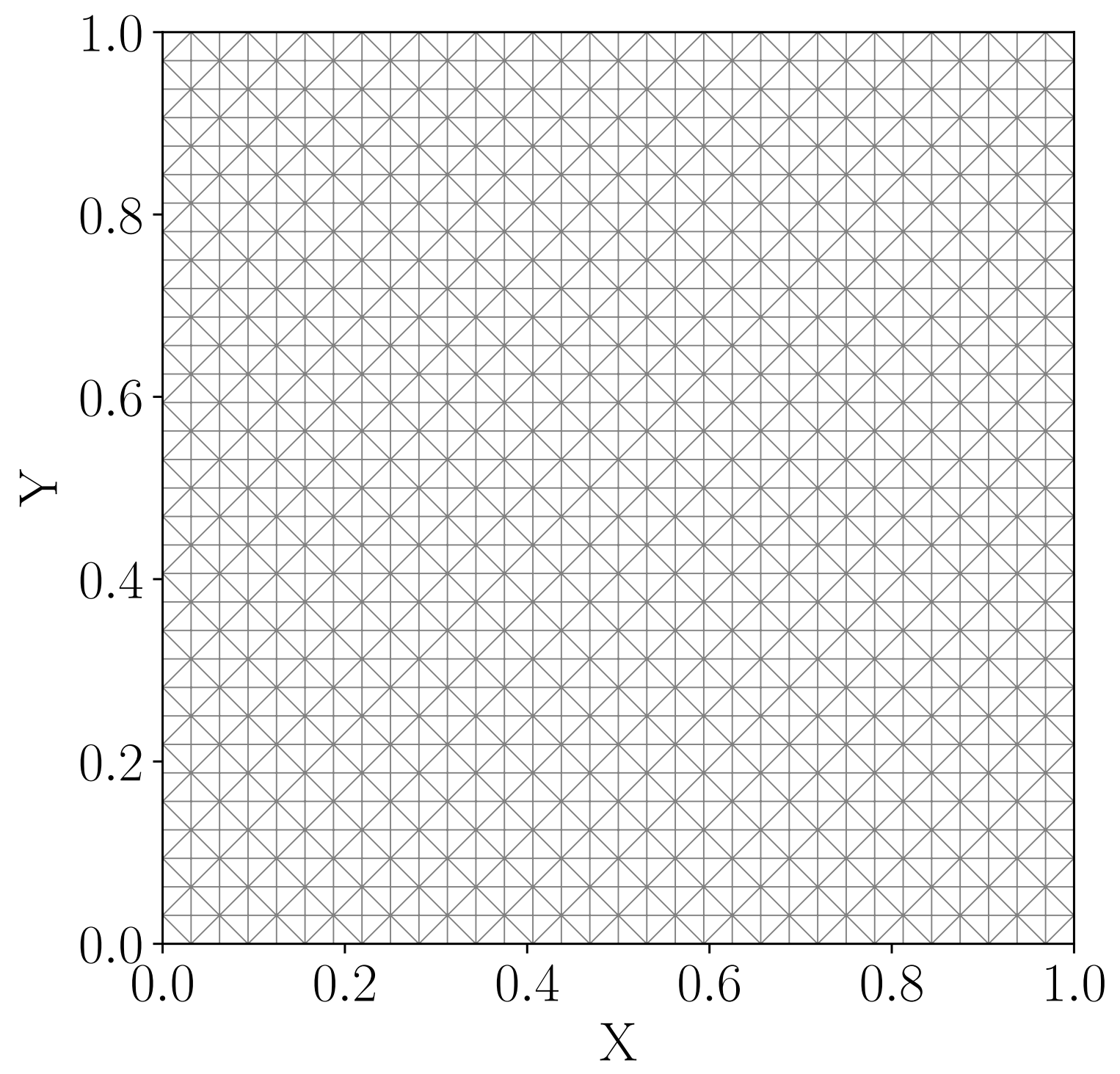

Figure S1. Example of triangular mesh used for computing the convergence rate of Fig. 4. We use the FENICS built-in mesh function, and cell size $h=1 / 32$. 

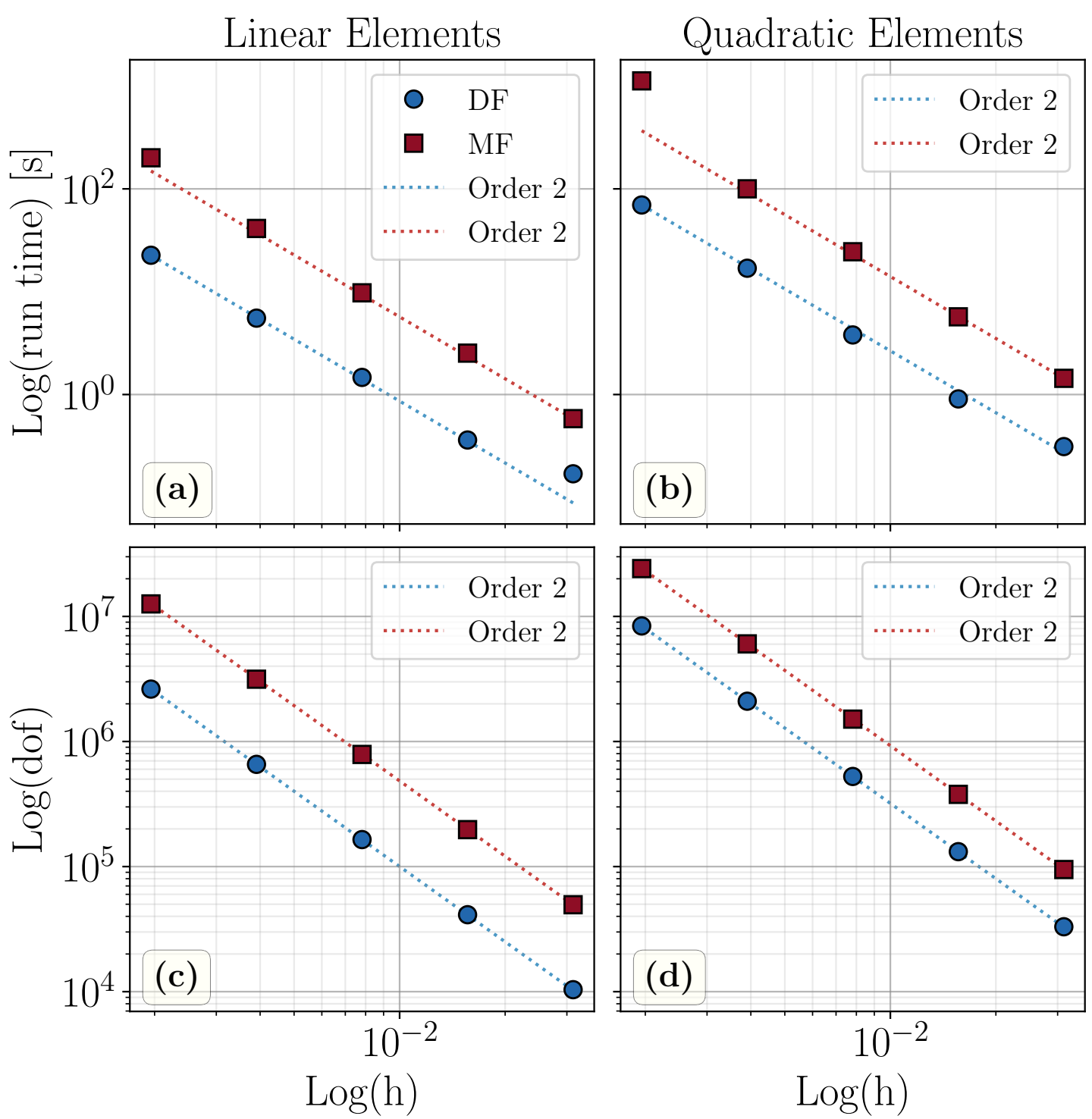

Figure S2. DOFs and computational time comparisons between the pure displacement formulation (DF, blue dots) and the mixed method (MF, red squares). Log-log plots of computational time (a-b) and DOFs (c-d) as a function of mesh size $h$ with linear and quadratic elements, respectively. Dashed lines in all plots indicate the best fit. 


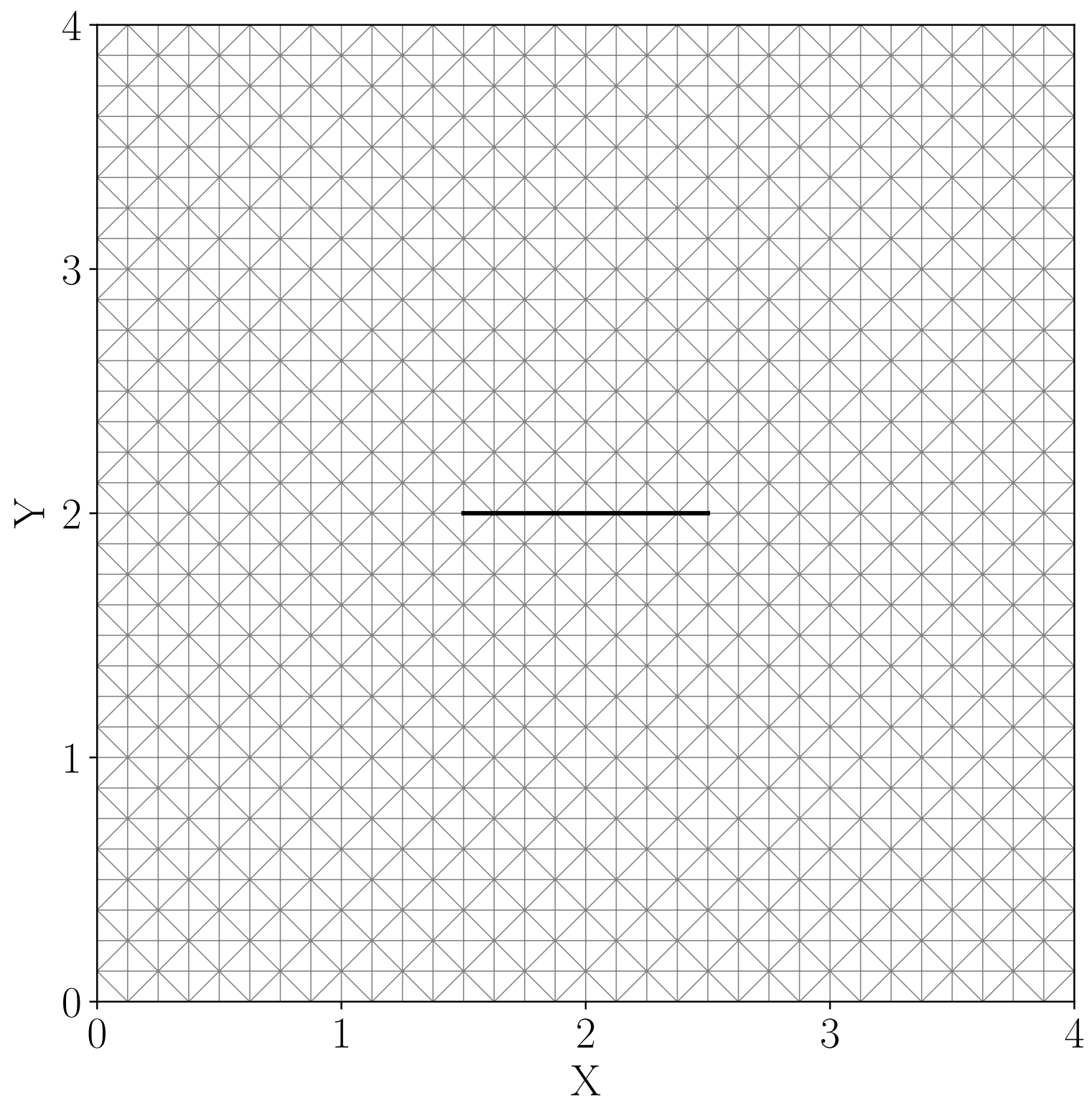

Figure S3. Example of triangular mesh used for computing the convergence rate of Fig. 6. We use the FENICS built-in mesh function. The black solid line indicates the crack, and cell size $h=1 / 8$. 

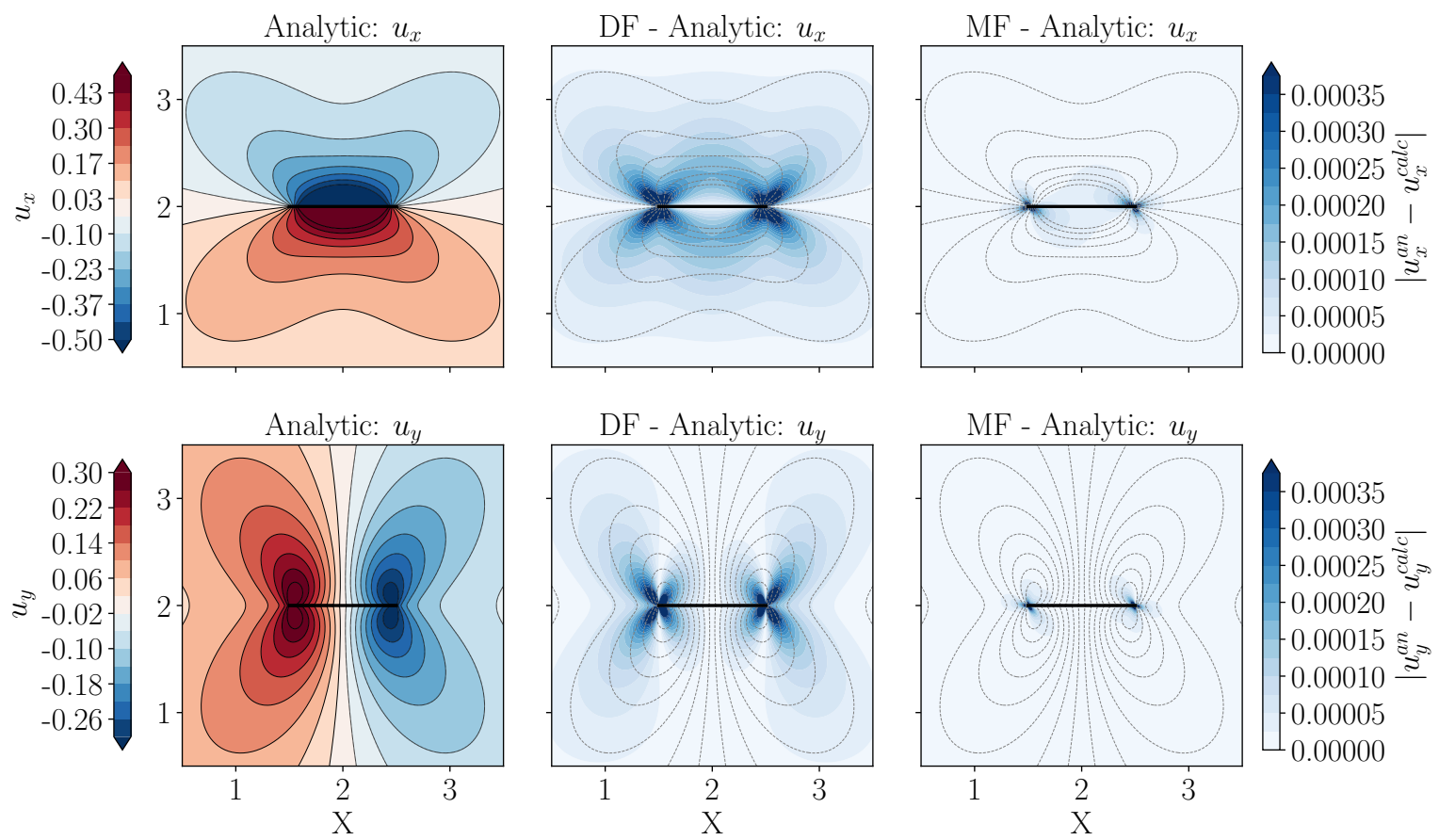

Figure S4. Comparison of the displacement components between the pure displacement formulation with the split node technique (DF), the mixed method with the fault implementation described in Section 3 (MF), and the analytic solution for a shear crack, mode II (Pollard \& Segall 1987). Top left to right: horizontal displacement of the analytic solution, and the absolute difference between DF and MF with respect to the analytic solution, respectively. Bottom left to right: same as above, but for the vertical displacement. Linear elements for displacement are used in all computations, and cell size $h=1 / 128$. The displacement components are normalized by the maximum analytic slip. 

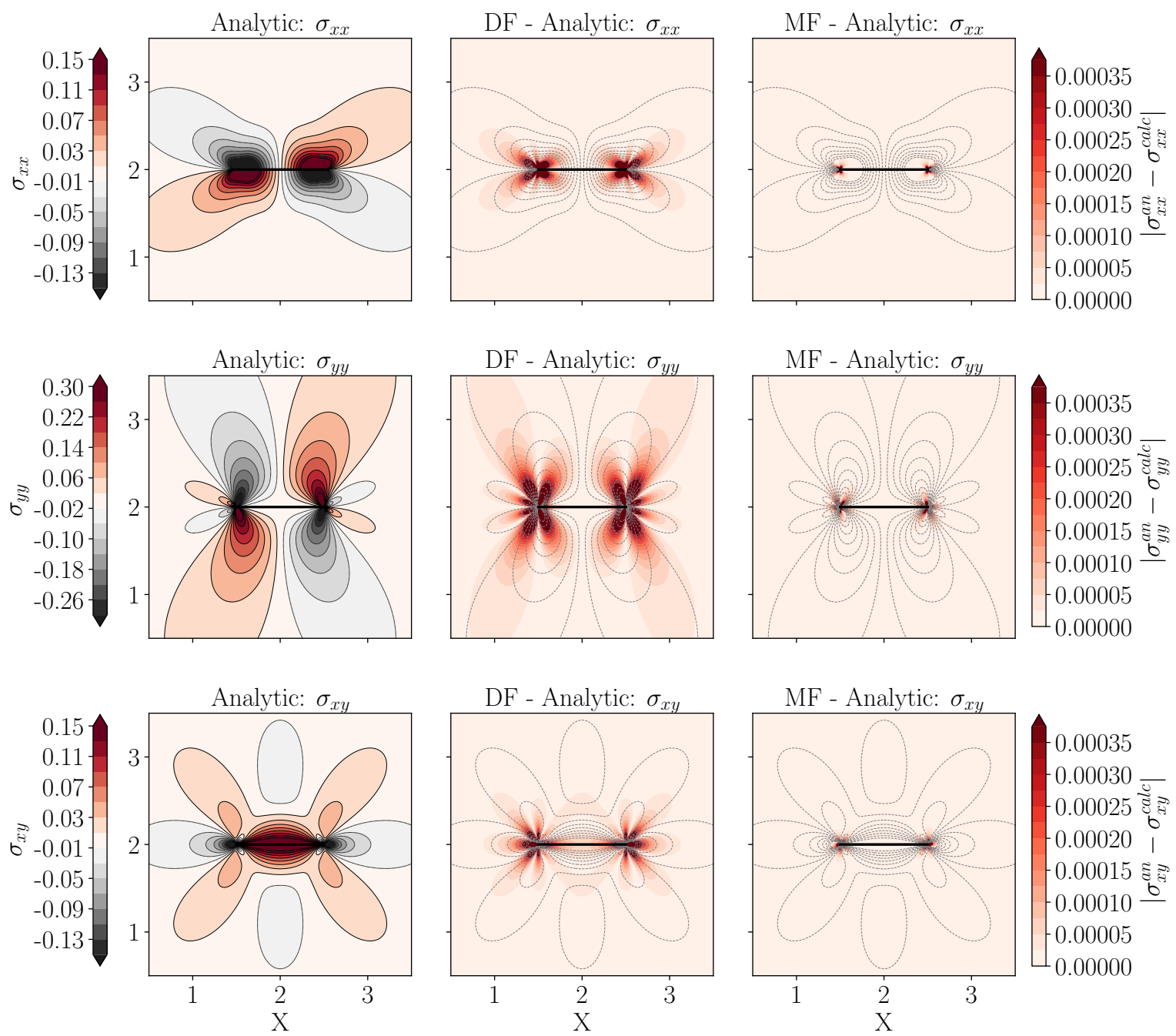

Figure S5. Comparison of the components of the stress tensor $\sigma$ between the pure displacement formulation with the split node technique (DF), the mixed method with the fault implementation described in Section 3 (MF), and the analytic solution for a shear crack, mode II (Pollard \& Segall 1987). Top left to right: $\sigma_{x x}$ of the analytic solution, and the absolute difference between DF and MF with respect to the analytic solution, respectively. Middle left to right: same as before, but for the $\sigma_{y y}$. Bottom left to right: same as above, but for the $\sigma_{x y}$. Linear elements for displacement are used in all computations, and cell size $h=1 / 128$. The stress components are normalized by the maximum analytic value. 

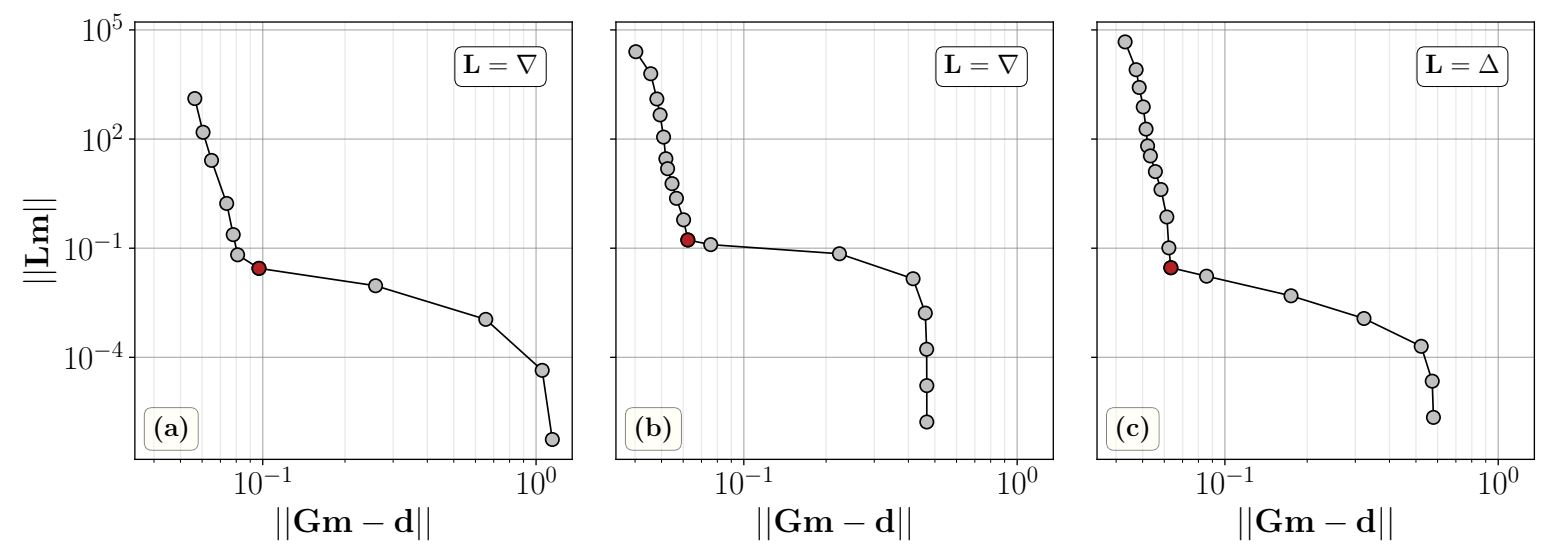

Figure S6. L-curve criterion for inferring the "optimal" regularization weight for the coseismic slip inversion. (a) L-curve log-log plot of the model norm as a function of the data norm for the adjoint inversion. $\mathbf{L}$ is a linear operator and in this case it is represented by the gradient. The "optimal" regularization weight is located a the "elbow" of the L-curve (red dot). (b)-(c) are same as (a) but for the standard linear inversion using the data kernel of Green's functions, and first-order and second-order Tikhonov regularization, respectively. In this case, $\mathbf{L}$ is represented by the gradient and Laplacian operator, respectively. The data are polluted with $5 \%$ random Gaussian noise with zero mean and covariance $\Gamma_{\text {noise }}$. 


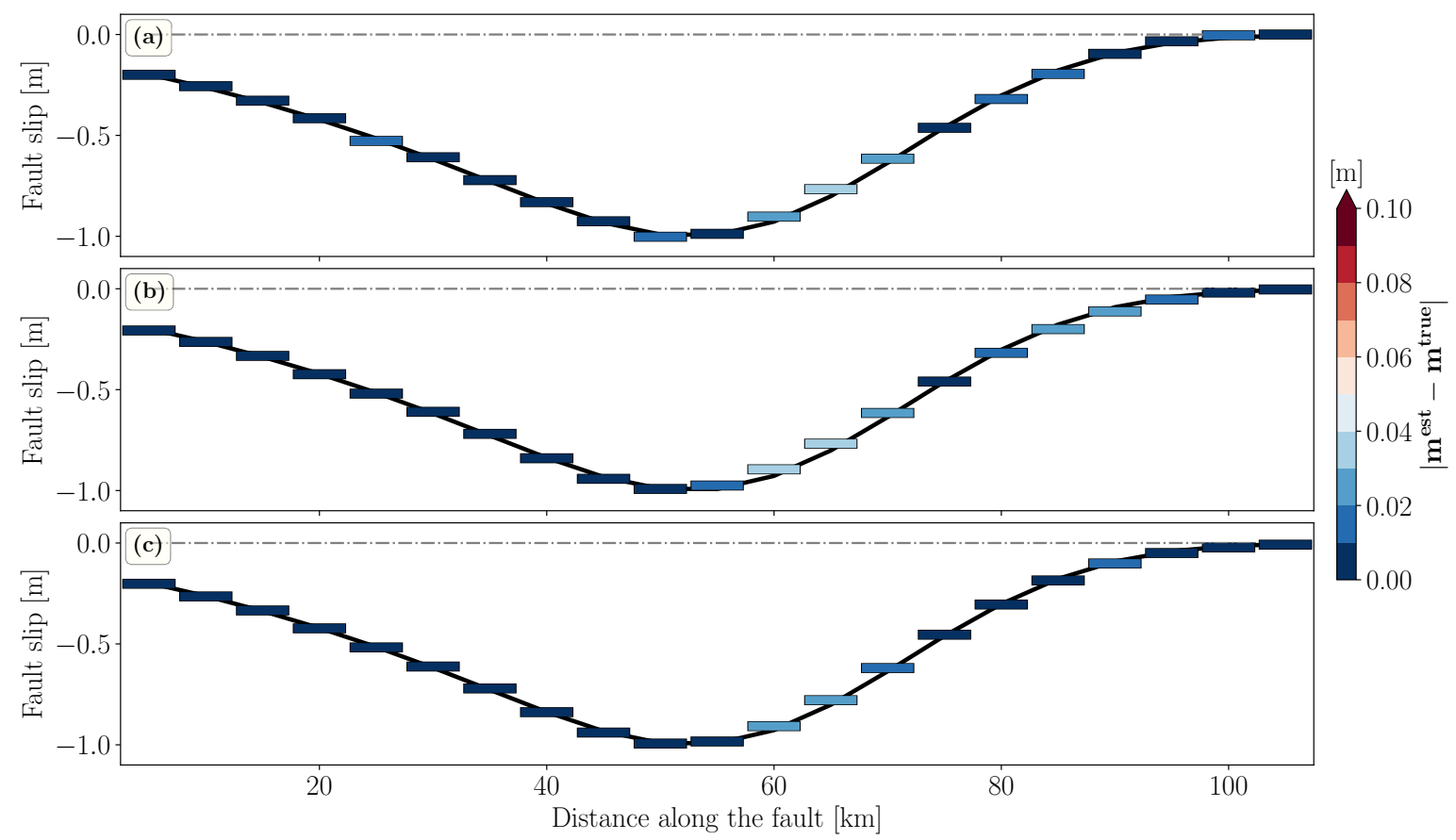

Figure S7. Reconstructed slip from the coseismic slip linear inversion. Same plots as Fig. 9, but for 1\% random Gaussian data noise. (a) Fault slip estimated using the new framework and the adjoint method. The slip is constant within each subfault patch (22 total), and it is represented by horizontal colored segments. Each slip segment is colored by the absolute error with respect to its true value. (b)-(c) show the same inversion results as (a) but using the standard linear approach with first-order (b) and second-order (c) Tikhonov regularization, respectively. 